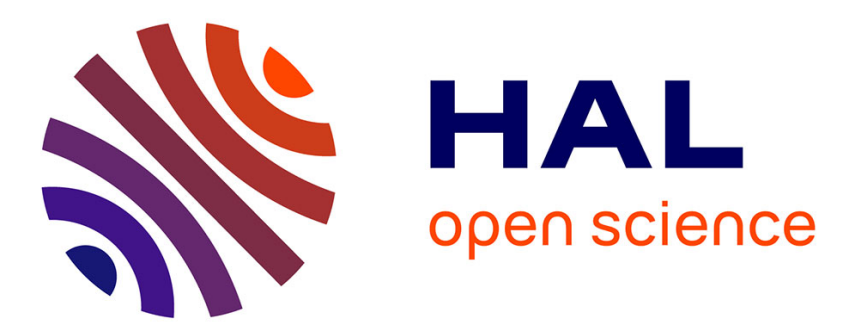

\title{
Large Deviation Principle for Non-Interacting Boson Random Point Processes
}

Hiroshi Tamura, Valentin A. Zagrebnov

\section{To cite this version:}

Hiroshi Tamura, Valentin A. Zagrebnov. Large Deviation Principle for Non-Interacting Boson Random Point Processes. Journal of Mathematical Physics, 2010, 51 (2), pp.023528 10.1063/1.3304115 . hal00413718

\section{HAL Id: hal-00413718 \\ https://hal.science/hal-00413718}

Submitted on 5 Sep 2009

HAL is a multi-disciplinary open access archive for the deposit and dissemination of scientific research documents, whether they are published or not. The documents may come from teaching and research institutions in France or abroad, or from public or private research centers.
L'archive ouverte pluridisciplinaire HAL, est destinée au dépôt et à la diffusion de documents scientifiques de niveau recherche, publiés ou non, émanant des établissements d'enseignement et de recherche français ou étrangers, des laboratoires publics ou privés. 


\title{
Large Deviation Principle for Non-Interacting Boson Random Point Processes
}

\author{
Hiroshi Tamura $]$ \\ Graduate School of the Natural Science and Technology \\ Kanazawa University, \\ Kanazawa 920-1192, Japan \\ Valentin A.Zagrebnov 2 \\ Université de la Méditerranée(Aix-Marseille II) and \\ Centre de Physique Théorique - UMR 6207 \\ Luminy-Case 907, 13288 Marseille Cedex 9, France
}

\begin{abstract}
Limit theorems, including the large deviation principle, are established for random point processes (fields), which describe the position distributions of the perfect boson gas in the regime of the Bose-Einstein condensation. We compare these results with those for the case of the normal phase.
\end{abstract}

Key words: Boson Random Point Processes, Bose-Einstein Condensation, Large Deviations, Central Limit Theorem

\footnotetext{
${ }^{1}$ tamurah@kenroku.kanazawa-u.ac.jp

${ }^{2}$ zagrebnov@cpt.univ-mrs.fr
} 


\section{Contents}

1 Introduction and Main Results 2

2 Preliminary arguments and general setting 4

3 Operators

4 Limit theorems for BEC 12

5 Conclusion 23

\section{$1 \quad$ Introduction and Main Results}

Fermion and boson random point processes (fields, or general Cox processus) were studied by many authors, in particular since they have a deep connection with the quantum statistical mechanics [TI1, TI3, TZ, F, Fr, FFr1, FFr2]. See also [S, Ly] and references therein. One of the advantages of the random point field approach to quantum statistical mechanical models is that it enables probabilistic limit theorems to apply to these models. In [ShTa], typical limit theorems are given for a certain class of random point processes which include the particular cases of the fermion as well as boson random point processes. In [TI2], the random point processes, which describe the position distribution of constituent particles of boson gases in Bose-Einstein Condensation (BEC) are constructed for the first time.

The purpose of the present paper is to give the limit theorems, such as the Law of the Large Numbers (LLN), the Central Limit Theorem (CLT) and the Large Deviation Principle (LDP) for the random boson point processes in the regime of the BEC. We compare them with the corresponding theorems for the normal phase (i.e. without the BEC). In the latter case a detailed study of the limit theorems, which do not use the random point processes formalism, is due to [LLS and GLM. In the last reference the authors consider even interacting quantum gases, but only in the rarified regime insuring the normal phase. These papers motivated the study of the large deviation principle in the Bogoliubov-type models [BZ], where BEC plays a key rôle in description of the model thermodynamic behaviour and the spectrum of excitations.

The study of the boson random point processes in the BEC regime is an interesting and delicate mathematical problem [TI2], see also a recent paper [E]. This last paper makes evident that a Cox process in the BEC regime [TI2] is driven by the square norm of a shifted Gaussian process. The shift is particle density dependent. In particular, this observation makes a contact with the Dynkin isomorphism theorem (known for Gaussian processus) as well as a relation between infinite devisability and factorisation of the boson Cox process involved in the BEC.

In the present paper, we study the limit theorems in the BEC regime ( Theorems 1.1, 1.2, 1.3) and discuss in Conclusion the comparison with the analogue of these Theorems in the normal phase. 
Let $\{G:=\exp (\beta \Delta)\}_{\beta \geq 0}$ be the (set-adjoint) heat semigroup generated by the Laplacian acting in $L^{2}\left(\mathbb{R}^{d}\right)$. For any non-negative bounded measurable function $f \geq 0$ with a compact support in $\mathbb{R}^{d}$, the operator

$$
W_{f}:=\left(G(1-G)^{-1}\right)^{1 / 2} \sqrt{1-e^{-f}},
$$

is a bounded and

$$
K_{f}:=W_{f}^{*} W_{f} \in \mathfrak{C}_{1}\left(L^{2}\left(\mathbb{R}^{d}\right)\right),
$$

i.e., is a trace-class operator on $L^{2}\left(\mathbb{R}^{d}\right)$. If $f=0$, then the operator $K:=K_{f=0}$ is bounded with the translation-invariant kernel:

$$
K(x, y)=\int_{\mathbb{R}^{d}} \frac{d p}{(2 \pi)^{d}} \frac{e^{i(x-y) p}}{e^{\beta|p|^{2}-1}} .
$$

Below we consider a noninteracting boson random point field $\nu_{\rho}$ for the total particle density $\rho>\rho_{c}$, which is characterized by the generating functional [TI2]:

$$
\int_{Q\left(\mathbb{R}^{d}\right)} e^{-\langle f, \xi\rangle} \nu_{\rho}(d \xi)=\frac{\exp \left(-\left(\rho-\rho_{c}\right)\left(\sqrt{1-e^{-f}},\left(1+K_{f}\right)^{-1} \sqrt{1-e^{-f}}\right)\right)}{\operatorname{Det}\left[1+K_{f}\right]} .
$$

Here $Q\left(\mathbb{R}^{d}\right)$ is the space of all point measures on $\mathbb{R}^{d}$, Det stands for the Fredholm determinant and $\langle f, \xi\rangle=\sum_{j} f\left(x_{j}\right)$, if $\xi=\sum_{j} \delta_{x_{j}} \in Q\left(\mathbb{R}^{d}\right)$.

The critical particle density, $\rho_{c}:=\rho_{c}(\beta)$, for the perfect Bose-gas can be expressed as:

$$
\rho_{c}(\beta):=K(x, x)=\int_{\mathbb{R}^{d}} \frac{d p}{(2 \pi)^{d}} \frac{e^{-\beta|p|^{2}}}{1-e^{-\beta|p|^{2}}}=\frac{\zeta(d / 2)}{(4 \pi \beta)^{d / 2}} .
$$

The random point field $\nu_{\rho}$ defined by by the generating functional (1.1) was introduced in [T12 to describe the Bose-Einstein condensation in the non-interacting (perfect) boson gas. For the detailed presentation of these notions, we refer to Ref. [TI2]. (See also the next Section 2.)

Below in the present paper, we use the following notations: $\|\cdot\|_{p}$ for $L^{p}\left(\mathbb{R}^{d}\right)$ norm and $\|\cdot\|$ for the bounded operators norm on $L^{2}\left(\mathbb{R}^{d}\right)$.

With these notations the main results of the paper can be expressed as follows:

Theorem 1.1 (Law of Large Numbers) For $\kappa \rightarrow \infty$, the limit

$$
\frac{1}{\kappa^{d}}\langle f(\cdot / \kappa), \xi\rangle \longrightarrow \rho \int_{\mathbb{R}^{d}} d x f(x)
$$

holds in $L^{2}\left(Q\left(\mathbb{R}^{d}\right), \nu_{\rho}\right)$.

Theorem 1.2 (Central Limit Theorem) Let

$$
Z_{\kappa}:=\frac{\langle f(\cdot / \kappa), \xi\rangle-\kappa^{d} \rho \int_{\mathbb{R}^{d}} d x f(x)}{\sqrt{2\left(\rho-\rho_{c}\right)}\left\|(-\beta \Delta)^{-1 / 2} f\right\|_{2} \kappa^{(d+2) / 2}} .
$$

Then the limit

$$
\lim _{\kappa \rightarrow \infty} \int_{Q\left(\mathbb{R}^{d}\right)} e^{i t Z_{\kappa}} \nu_{\rho}(d \xi)=e^{-t^{2} / 2}
$$


Theorem 1.3 (Large Deviation Principle) There exists a certain (bona fide) rate convex function $I: \mathbb{R} \mapsto[0,+\infty]$, such that the limits

$$
\limsup _{\kappa \rightarrow \infty} \frac{1}{\kappa^{d-2}} \log \nu_{\rho}\left(\frac{1}{\kappa^{d}}\langle f(\cdot / \kappa), \xi\rangle \in F\right) \leqslant-\inf _{s \in F} I(s) \quad \text { for any closed } F \subset \mathbb{R},
$$

and

$$
\liminf _{\kappa \rightarrow \infty} \frac{1}{\kappa^{d-2}} \log \nu_{\rho}\left(\frac{1}{\kappa^{d}}\langle f(\cdot / \kappa), \xi\rangle \in G\right) \geqslant-\inf _{s \in G} I(s) \quad \text { for any open } G \subset \mathbb{R},
$$

hold.

In Section 5 we compare these results with those for boson random point processes in the normal phase.

\section{Preliminary arguments and general setting}

Let $R$ be a locally compact Hausdorff space with countable basis, and $\lambda$ be a positive Radon measure on $R$. We suppose that the non-negative (possibly unbounded) selfadjoint operator $K$ in $L^{2}(R, \lambda)$ satisfies the following condition $\mathbf{K}$ '.

\section{Condition $\mathrm{K}^{\prime}$ :}

(i) (locally trace class) For every bounded Borel set $\Lambda \subset R, K^{1 / 2} \chi_{\Lambda}$ is a Hilbert-Schmidt operator, where $\chi_{\Lambda}$ denotes the multiplication operator corresponding to the indicator function of the set $\Lambda$, which we denote by the same symbol.

(ii) The operator $G=K(1+K)^{-1}$ has non-negative integral kernel $G(x, y)$, which satisfies the conditions:

$$
\begin{array}{cl}
G(x, y)>0 & \lambda \otimes \lambda-\text { a.e. }(x, y) \in R^{2}, \\
\int_{R} G(x, y) \lambda(d y) \leqslant 1 & \lambda-\text { a.e. } x \in R .
\end{array}
$$

The above conditions are arranged in such a way that one can simultaneously deal with the random point processes $\mu_{K}^{\text {det }}$ and $\mu_{K, \rho}$, see [TI2 and ShTa. In particular, the operator $K$ has a positive kernel $K(x, y)$, i.e.,

$$
K(x, y)>0 \quad \lambda \otimes \lambda-\text { a.e. }(x, y) \in R^{2},
$$

see [II2]. The operator $K_{\Lambda}:=\left(K^{1 / 2} \chi_{\Lambda}\right)^{*} K^{1 / 2} \chi_{\Lambda}$ is a trace-class operator. For a bounded measurable function $f$ with compact support, we define the operator

$$
K_{f}:=\sqrt{1-e^{-f}} K_{\Lambda} \sqrt{1-e^{-f}}
$$

where supp $f \subset \Lambda$. Note that $K_{f}$ is independent of the choice of $\Lambda$, which contains $\operatorname{supp} f$.

Let $Q(R)$ be Polish space of all locally finite non-negative integer-valued Borel measures on $R$. Recall that the Borel probability measures on $Q(R)$ (i.e. random point 
processes on $R$ ) $\mu_{K}^{(\text {det })}$ and $\mu_{K, \rho}$ are introduced in [ShTa, TI2] for $\rho>0$ by means of generating functionals:

$$
\begin{gathered}
\int_{Q(R)} e^{-\langle f, \xi\rangle} \mu_{K}^{(d e t)}(d \xi)=\operatorname{Det}\left[1+K_{f}\right]^{-1}=\operatorname{Det}\left[1+\left(1-e^{-f}\right) K_{\Lambda}\right]^{-1} \\
\int_{Q(R)} e^{-\langle f, \xi\rangle} \mu_{K, \rho}(d \xi)=\exp \left\{-\rho\left\langle\sqrt{1-e^{-f}},\left(1+K_{f}\right)^{-1} \sqrt{1-e^{-f}}\right\rangle\right\} \\
=\exp \left\{-\rho\left\langle\chi_{\Lambda},\left(1+\left(1-e^{-f}\right) K_{\Lambda}\right)^{-1}\left(1-e^{-f}\right)\right\rangle\right\}
\end{gathered}
$$

for any function $f$ with $\Lambda \supset \operatorname{supp} f$.

It was shown [TI2 that for $R=\mathbb{R}^{d}$ the boson random point processes corresponding to the ideal Bose-gas in the regime of Bose-Einstein condensation $\left(\rho>\rho_{c}\right)$ is described by the convolution $\nu_{\rho}:=\mu_{K}^{\text {det }} * \mu_{K, \rho-\rho_{c}}$.

Theorem 2.1 For any non-negative bounded measurable function $f$ on $R$ with compact support supp $f \subset \Lambda$ in a bounded Borel set $\Lambda \subset R$ one has the following equalities:

$$
\begin{aligned}
& \int_{Q(R)} e^{i\langle f, \xi\rangle} \mu_{K, \rho}(d \xi)=\exp \left[-\rho\left\langle\chi_{\Lambda},\left(1+\left(1-e^{i f}\right) K_{\Lambda}\right)^{-1}\left(1-e^{i f}\right)\right\rangle\right], \\
& \int_{Q(R)} e^{\langle f, \xi\rangle} \mu_{K, \rho}(d \xi)=\left\{\begin{array}{rr}
\exp \left[\rho\left\langle\sqrt{e^{f}-1},\left(1-\sqrt{e^{f}-1} K_{\Lambda} \sqrt{e^{f}-1}\right)^{-1} \sqrt{e^{f}-1}\right\rangle\right]<\infty \\
\infty & \text { for }\left\|\sqrt{e^{f}-1} K_{\Lambda} \sqrt{e^{f}-1}\right\|<1 \\
& \text { for }\left\|\sqrt{e^{f}-1} K_{\Lambda} \sqrt{e^{f}-1}\right\| \geqslant 1,
\end{array}\right.
\end{aligned}
$$

$$
\begin{aligned}
& \int_{Q(R)} e^{i\langle f, \xi\rangle} \mu_{K}^{(d e t)}(d \xi)=\operatorname{Det}\left[1+\left(1-e^{i f}\right) K_{\Lambda}\right]^{-1},
\end{aligned}
$$

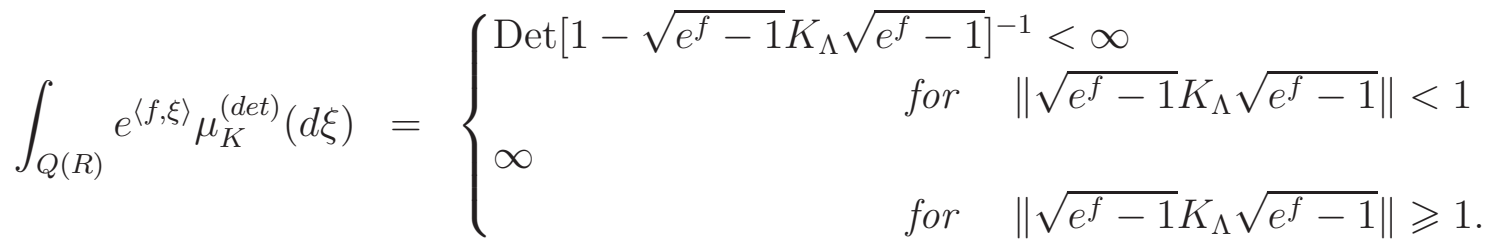

Proof: Let $f \not \equiv 0$, i.e., $\lambda(\operatorname{supp} f)>0$. In [TI2], pp.213-214, it was introduced a family of symmetric non-negative functions $\left\{\sigma_{\Lambda^{n}}\right\}_{n \geq 0}$ defined by the equations:

$$
\begin{gathered}
\exp \left[-\rho\left\langle\sqrt{1-e^{-f}},\left(1+K_{f}\right)^{-1} \sqrt{1-e^{-f}}\right\rangle\right]=\exp \left[-\rho\left\langle\chi_{\Lambda},\left(1+\left(1-e^{-f}\right) K_{\Lambda}\right)^{-1}\left(1-e^{-f}\right)\right\rangle\right] \\
=\exp \left[-\rho\left\langle\chi_{\Lambda},\left(1+K_{\Lambda}\right)^{-1} \chi_{\Lambda}\right\rangle+\rho \sum_{l=0}^{\infty}\left\langle\left(1+K_{\Lambda}\right)^{-1} \chi_{\Lambda}, e^{-f}\left(R_{\Lambda} e^{-f}\right)^{l}\left(1+K_{\Lambda}\right)^{-1} \chi_{\Lambda}\right\rangle\right] \\
=\sum_{n=0}^{\infty} \frac{1}{n !} \int_{\Lambda^{n}} \sigma_{\Lambda^{n}}\left(x_{1}, \cdots, x_{n}\right) e^{-\sum_{k=1}^{n} f\left(x_{k}\right)} \lambda^{\otimes n}\left(d x_{1} \cdots d x_{n}\right) .
\end{gathered}
$$


Here, $R_{\Lambda}=K_{\Lambda}\left(1+K_{\Lambda}\right)^{-1}$ satisfies $\left\|R_{\Lambda}\right\|<1$ since $K_{\Lambda}$ is a bounded non-negative operator. Using $\left\{\sigma_{\Lambda^{n}}\right\}_{n \geq 0}$, the random point processes $\mu_{K, \rho}$ was defined as the probability measure such that

$$
\int_{Q(R)} F(\xi) \mu_{K, \rho}(d \xi)=\sum_{n=0}^{\infty} \int_{\Lambda^{n}} \sigma_{\Lambda^{n}}\left(x_{1}, \cdots, x_{n}\right) F\left(\sum_{j=1}^{n} \delta_{x_{j}}\right) \lambda^{\otimes n}\left(d x_{1}, \cdots, d x_{n}\right)
$$

holds for any bounded (or non-negative) measurable functional satisfying $F(\xi)=F\left(\xi_{\Lambda}\right)$, where $\xi_{\Lambda}(A)=\xi(A \cap \Lambda)$.

From this construction, we obtain the first claim (1):

$$
\begin{gathered}
\int_{Q(R)} e^{i\langle f, \xi\rangle} \mu_{K, \rho}(d \xi)=\sum_{n=0}^{\infty} \int_{\Lambda^{n}} \sigma_{\Lambda^{n}}\left(x_{1}, \cdots, x_{n}\right) e^{i \sum_{j=1}^{n} f\left(x_{j}\right)} \lambda^{\otimes n}\left(d x_{1}, \cdots, d x_{n}\right) \\
=\exp \left[-\rho\left\langle\chi_{\Lambda},\left(1+K_{\Lambda}\right)^{-1} \chi_{\Lambda}\right\rangle+\rho \sum_{l=0}^{\infty}\left\langle\left(1+K_{\Lambda}\right)^{-1} \chi_{\Lambda}, e^{i f}\left(R_{\Lambda} e^{i f}\right)^{l}\left(1+K_{\Lambda}\right)^{-1} \chi_{\Lambda}\right\rangle\right] \\
=\exp \left[-\rho\left\langle\chi_{\Lambda},\left(1+\left(1-e^{i f}\right) K_{\Lambda}\right)^{-1}\left(1-e^{i f}\right)\right\rangle\right] .
\end{gathered}
$$

If $z \in \mathbb{C}$ satisfies $|z| e^{\|f\|_{\infty}} \leqslant 1$, then we get the equality:

$$
\begin{gathered}
\sum_{n=0}^{\infty} \int_{\Lambda^{n}} \sigma_{\Lambda^{n}}\left(x_{1}, \cdots, x_{n}\right) z^{n} e^{\sum_{j=1}^{n} f\left(x_{j}\right)} \lambda^{\otimes n}\left(d x_{1}, \cdots, d x_{n}\right) \\
=\exp \left[-\rho\left\langle\chi_{\Lambda},\left(1+K_{\Lambda}\right)^{-1} \chi_{\Lambda}\right\rangle+\rho \sum_{l=0}^{\infty} z^{l+1}\left\langle\left(1+K_{\Lambda}\right)^{-1} \chi_{\Lambda}, e^{f}\left(R_{\Lambda} e^{f}\right)^{l}\left(1+K_{\Lambda}\right)^{-1} \chi_{\Lambda}\right\rangle\right],
\end{gathered}
$$

Since in the both sides all coefficients the $z$-power series are non-negative, this equality (2.5) also holds for $z=1$ in the sense that either the both sides are finite and equal or they are both diverge to $+\infty$. When they are finite, we obtain

$$
\int_{Q(R)} e^{\langle f, \xi\rangle} \mu_{K, \rho}(d \xi)=\exp \left[\rho\left\langle\sqrt{e^{f}-1},\left(1-\sqrt{e^{f}-1} K_{\Lambda} \sqrt{e^{f}-1}\right)^{-1} \sqrt{e^{f}-1}\right\rangle\right],
$$

cf. the proof of Theorem 2.1 in [T12], pp.213-214. Hence, for the second claim (2) it is sufficient to show that

$$
\text { the finite RHS of } 2.5) \Leftrightarrow\left\|e^{f / 2} R_{\Lambda} e^{f / 2}\right\|<1 \Leftrightarrow\left\|\sqrt{e^{f}-1} K_{\Lambda} \sqrt{e^{f}-1}\right\|<1 \text {. }
$$

Notice that by Proposition 2.3(ii) [TI2] the Condition $\mathbf{K}^{\prime}$ (ii) ensures: $R_{\Lambda}(x, y)>0$, for $\lambda \otimes \lambda$-almost all $(x, y) \in \Lambda^{2}$. Since $R_{\Lambda}$ is a compact symmetric operator, it follows from the variational principle that $\left\|e^{f / 2} R_{\Lambda} e^{f / 2}\right\|$ is the largest eigenvalue of the operator $e^{f / 2} R_{\Lambda} e^{f / 2}$ with eigenfunction $\varphi_{0}>0(\lambda-a . e$. on $\Lambda)$. Hence we have

$$
\begin{gathered}
\left\langle\left(1+K_{\Lambda}\right)^{-1} \chi_{\Lambda}, e^{f}\left(R_{\Lambda} e^{f}\right)^{l}\left(1+K_{\Lambda}\right)^{-1} \chi_{\Lambda}\right\rangle \\
=\left|\left\langle\varphi_{0}, e^{f / 2}\left(1+K_{\Lambda}\right)^{-1} \chi_{\Lambda}\right\rangle\right|^{2}\left\|e^{f / 2} R_{\Lambda} e^{f / 2}\right\|^{l}+O\left(\left\|e^{f / 2} R_{\Lambda} e^{f / 2}\right\|^{l} \delta^{l}\right)
\end{gathered}
$$


for some $\delta \in(0,1)$. Note that $\left|\left\langle\varphi_{0}, e^{f / 2}\left(1+K_{\Lambda}\right)^{-1} \chi_{\Lambda}\right\rangle\right|>0$ because $\left(1+K_{\Lambda}\right)^{-1} \chi_{\Lambda}>$ $0(\lambda$-a.e. on $\Lambda)$ and $\left\|\left(1+K_{\Lambda}\right)^{-1} \chi_{\Lambda}\right\|>0$. Thus, we get the first equivalence in (2.6).

For the second equivalence, it is enough to prove that

$$
\left\|R_{\Lambda}^{1 / 2} e^{f} R_{\Lambda}^{1 / 2}\right\|<1 \Longleftrightarrow\left\|K_{\Lambda}^{1 / 2}\left(e^{f}-1\right) K_{\Lambda}^{1 / 2}\right\|<1
$$

by duality. Let $\left\|R_{\Lambda}^{1 / 2} e^{f} R_{\Lambda}^{1 / 2}\right\|=\eta<1$. Then $K_{\Lambda} \geqslant 0, f \geqslant 0$ and

$$
1-K_{\Lambda}^{1 / 2}\left(e^{f}-1\right) K_{\Lambda}^{1 / 2}=\left(1+K_{\Lambda}\right)^{1 / 2}\left(1-R_{\Lambda}^{1 / 2} e^{f} R_{\Lambda}^{1 / 2}\right)\left(1+K_{\Lambda}\right)^{1 / 2},
$$

together with $R_{\Lambda}^{1 / 2} e^{f} R_{\Lambda}^{1 / 2} \geq 0$, imply

$$
1-K_{\Lambda}^{1 / 2}\left(e^{f}-1\right) K_{\Lambda}^{1 / 2} \geqslant\left(1+K_{\Lambda}\right)(1-\eta) \geqslant 1-\eta .
$$

Hence $K_{\Lambda}^{1 / 2}\left(e^{f}-1\right) K_{\Lambda}^{1 / 2} \leqslant \eta<1$. On the other hand, if $\left\|K_{\Lambda}^{1 / 2}\left(e^{f}-1\right) K_{\Lambda}^{1 / 2}\right\|=\theta<1$, then

$$
\begin{aligned}
1-R_{\Lambda}^{1 / 2} e^{f} R_{\Lambda}^{1 / 2} & =\left(1+K_{\Lambda}\right)^{-1 / 2}\left(1-K_{\Lambda}^{1 / 2}\left(e^{f}-1\right) K_{\Lambda}^{1 / 2}\right)\left(1+K_{\Lambda}\right)^{-1 / 2} \\
& \geqslant(1-\theta)\left(1+K_{\Lambda}\right)^{-1} \geqslant \frac{1-\theta}{1+\left\|K_{\Lambda}\right\|},
\end{aligned}
$$

which yields

$$
0 \leqslant R_{\Lambda}^{1 / 2} e^{f} R_{\Lambda}^{1 / 2} \leqslant 1-\frac{1-\theta}{1+\left\|K_{\Lambda}\right\|}<1
$$

This finishes the proof of claims (1) and (2) of the Theorem concerning the measure $\mu_{K, \rho}$.

The claims (3) and (4) concerning the measure $\mu_{K}^{(d e t)}$ can be shown similarly if one uses, instead of (2.3), the representation:

$$
\begin{gathered}
\operatorname{Det}\left[1+K_{f}\right]^{-1}=\operatorname{Det}\left[1+K_{\Lambda}\right]^{-1} \operatorname{Det}\left[1-e^{-f} R_{\Lambda}\right]^{-1} \\
=\operatorname{Det}\left[1+K_{\Lambda}\right]^{-1} \sum_{n=0}^{\infty} \frac{1}{n !} \int_{R^{n}} \operatorname{Per}\left\{R_{\Lambda}\left(x_{j}, x_{k}\right)\right\}_{1 \leqslant j, k \leqslant n} e^{-\sum_{l=1}^{n} f\left(x_{l}\right)} \lambda^{\otimes n}\left(d x_{1}, \cdots, d x_{n}\right),
\end{gathered}
$$

where Det is the Fredholm determinant and Per is the permanent of the corresponding matrices ShTa.

\section{Operators}

Below we deal with the boson random point processes which describe the position distribution of the perfect Bose-gas $\left(\mathbb{R}^{d}\right.$ for $d>2$ ) above the critical particle density $\rho_{c}:=\rho_{c}(\beta)$, i.e. in the regime of the Bose-Einstein condensation.

To this end we set $R:=\mathbb{R}^{d}$ and $K^{\beta}:=G^{\beta}\left(1-G^{\beta}\right)^{-1}$ for $K$, where $G^{\beta}:=e^{\beta \Delta}$ for $G$. Here $\beta>0$ is the inverse temperature and $\Delta$ denotes the $d$-dimensional self-adjoint Laplacian operator in the space $L^{2}\left(\mathbb{R}^{d}\right)$ equipped by the Lebesgue measure. Then it can be shown that operator $K^{\beta}$ satisfies the Condition $\mathbf{K}^{\prime}$, see [TI2]. 
In the present section, we derive some miscellaneous properties of the operators, which we use in the line of reasoning of the next section. First we adopt the following definition of the Fourier transformation:

$$
\widetilde{h}(p):=(\mathscr{F} h)(p)=\int_{\mathbb{R}^{d}} e^{-i p \cdot x} h(x) \frac{d x}{(2 \pi)^{d / 2}}
$$

for $h \in L^{1}\left(\mathbb{R}^{d}\right)$ and for its extension to $L^{2}\left(\mathbb{R}^{d}\right)$.

Lemma 3.1 For any compact $\Lambda \subset \mathbb{R}^{d}$ the operator $(-\Delta)^{-1 / 2} \chi_{\Lambda},\left(K^{\beta}\right)^{1 / 2} \chi_{\Lambda}$ is bounded. Therefore,

$$
\begin{gathered}
(-\Delta)_{\Lambda}^{-1}:=\left((-\Delta)^{-1 / 2} \chi_{\Lambda}\right)^{*}(-\Delta)^{-1 / 2} \chi_{\Lambda} \\
K_{\Lambda}^{\beta}:=\left(\left(K^{\beta}\right)^{-1 / 2} \chi_{\Lambda}\right)^{*}\left(K^{\beta}\right)^{-1 / 2} \chi_{\Lambda}
\end{gathered}
$$

are bounded non-negative self-adjoint operators.

Proof: These properties can be verified with a help of the Fourier transformation. For any $g \in L^{2}\left(\mathbb{R}^{d}\right)$, we obtain:

$$
\begin{gathered}
\left\|(-\Delta)^{-1 / 2} \chi_{\Lambda} g\right\|_{2}^{2}=\int_{\mathbb{R}^{d}} \frac{\left|\widetilde{\chi_{\Lambda} g}(p)\right|^{2}}{|p|^{2}} d p \\
\leqslant \int_{|p| \leqslant 1} \frac{\left\|\widetilde{\chi_{\Lambda} g}\right\|_{\infty}^{2}}{|p|^{2}} d p+\int_{\mathbb{R}^{d}}\left|\widetilde{\chi_{\Lambda} g}(p)\right|^{2} d p \\
\leqslant c_{1}\left\|\chi_{\Lambda} g\right\|_{1}^{2}+\left\|\chi_{\Lambda} g\right\|_{2}^{2} \leqslant c_{2}\left\|\chi_{\Lambda}\right\|_{2}^{2}\|g\|_{2}^{2}+\left\|\chi_{\Lambda}\right\|_{\infty}^{2}\|g\|_{2}^{2} \\
=(1+c|\Lambda|)\|g\|_{2}^{2} .
\end{gathered}
$$

Thus, $(-\Delta)^{-1 / 2} \chi_{\Lambda}$ is bounded and $\left\|(-\Delta)^{-1 / 2} \chi_{\Lambda}\right\| \leqslant \sqrt{1+c|\Lambda|}$ holds. It gives $\left\|(-\Delta)_{\Lambda}^{-1}\right\| \leqslant$ $1+c|\Lambda|$. Here, $|\Lambda|$ denotes the Lebesgue measure of $\Lambda$.

A similar argument is valid for the operator $K_{\Lambda}^{\beta}$.

Definition 3.2 For $\kappa>0$, we define the transformation

$$
U_{\kappa}: L^{2}\left(\mathbb{R}^{d}\right) \ni g(\cdot) \mapsto \kappa^{d / 2} g(\kappa \cdot) \in L^{2}\left(\mathbb{R}^{d}\right) .
$$

Lemma 3.3 The transformation $U_{\kappa}$ is unitary on $L^{2}\left(\mathbb{R}^{d}\right)$ for any $\kappa>0$, and it has the following properties:

(1) $\quad U_{\kappa} h U_{\kappa}^{-1}=h(\kappa \cdot) \quad$ for the multiplication operator by function $h$.

(2) $U_{\kappa} \Delta U_{\kappa}^{-1}=\kappa^{-2} \Delta$.

(3) $U_{\kappa}(-\Delta)_{\kappa \Lambda}^{-1} U_{\kappa}^{-1}=\kappa^{2}(-\Delta)_{\Lambda}^{-1}, \quad U_{\kappa} G^{\beta} U_{\kappa}^{-1}=G^{\beta / \kappa^{2}}$.

(4) $U_{\kappa} K_{\kappa \Lambda}^{\beta} U_{\kappa}^{-1}=K_{\Lambda}^{\beta / \kappa^{2}}$.

Proof: These properties are a straightforward consequence of the relation $\mathscr{F} U_{\kappa}=U_{\kappa}^{-1} \mathscr{F}$ on $L^{2}\left(\mathbb{R}^{d}\right)$. 
Definition 3.4 For bounded non-negative function $f$ with a compact support and for $\kappa>0$, we put

$$
f_{\kappa}^{( \pm)}(x):= \pm \kappa^{2}\left(e^{ \pm f(x) / \kappa^{2}}-1\right)
$$

Lemma 3.5 One has the following estimates:

$$
\begin{gathered}
f_{\kappa}^{( \pm)}(x) \geqslant 0, \quad\left|\chi_{\{f>0\}}(x) \frac{f_{\kappa}^{( \pm)}(x)}{f(x)}\right| \leqslant e^{\|f\|_{\infty} / \kappa^{2}}, \quad \chi_{\{f>0\}}(x)\left|1-\sqrt{\frac{f_{\kappa}^{( \pm)}(x)}{f(x)}}\right| \leqslant \frac{\|f\|_{\infty}}{2 \kappa^{2}} e^{\|f\|_{\infty} / \kappa^{2}}, \\
\left\|f_{\kappa}^{( \pm)}\right\|_{\infty} \leqslant\|f\|_{\infty} e^{\|f\|_{\infty} / \kappa^{2}}, \quad\left\|f-f_{\kappa}^{( \pm)}\right\|_{\infty} \leqslant \frac{\|f\|_{\infty}}{2 \kappa^{2}} e^{\|f\|_{\infty} / \kappa^{2}} .
\end{gathered}
$$

Proof: These estimates are a direct consequence of the elementary inequalities:

$$
\frac{\left|e^{y}-1\right|}{|y|} \leqslant e^{|y|}, \quad \frac{\left|e^{y}-1-y\right|}{|y|} \leqslant \frac{|y| e^{|y|}}{2}
$$

and $|\sqrt{z}-1| \leqslant|z-1|$ for $y \in \mathbb{R}-\{0\}, z \geqslant 0$.

Lemma 3.6 For any $\kappa>0$ we have the estimates:

$$
0 \leqslant(-\beta \Delta)_{\Lambda}^{-1}-\kappa^{-2} K_{\Lambda}^{\beta / \kappa^{2}} \leqslant\left(2 \kappa^{2}\right)^{-1}
$$

Proof: Using the Fourier transformation, we get

$$
\left\langle g,\left[\kappa^{2}(-\beta \Delta)_{\Lambda}^{-1}-K_{\Lambda}^{\beta / \kappa^{2}}\right] g\right\rangle=\int_{\mathbb{R}^{d}}\left(\frac{\kappa^{2}}{\beta|p|^{2}}-\frac{1}{e^{\beta|p|^{2} / \kappa^{2}-1}}\right)\left|\widetilde{\chi_{\Lambda} g}(p)\right|^{2} d p .
$$

Then lemma follows from the inequality

$$
0 \leqslant \frac{1}{y}-\frac{1}{e^{y}-1} \leqslant \frac{1}{2}, \quad \text { for } y>0
$$

and from the estimate $\left\|\widetilde{\chi_{\Lambda} g}\right\|_{2}=\left\|\chi_{\Lambda} g\right\|_{2} \leqslant\|g\|_{2}$.

Lemma 3.7 Suppose that $\operatorname{supp} f \subset \Lambda$. Then for $\kappa \rightarrow 0$ one gets the operator-norm asymptotics:

$$
\left\|\sqrt{f}(-\beta \Delta)_{\Lambda}^{-1} \sqrt{f}-\kappa^{-2} \sqrt{f_{\kappa}^{( \pm)}} K_{\Lambda}^{\beta / \kappa^{2}} \sqrt{f_{\kappa}^{( \pm)}}\right\|=O\left(\kappa^{-2}\right)
$$

in the space $L^{2}\left(\mathbb{R}^{d}\right)$. 
Proof : From Lemma 3.1, 3.5 and 3.6, we obtain

$$
\begin{gathered}
\left\|\sqrt{f}(-\beta \Delta)_{\Lambda}^{-1} \sqrt{f}-\kappa^{-2} \sqrt{f_{\kappa}^{( \pm)}} K_{\Lambda}^{\beta / \kappa^{2}} \sqrt{f_{\kappa}^{( \pm)}}\right\| \\
\leqslant\left\|\left(\sqrt{f}-\sqrt{f_{\kappa}^{( \pm)}}\right)(-\beta \Delta)_{\Lambda}^{-1} \sqrt{f}\right\|+\left\|\sqrt{f_{\kappa}^{( \pm)}}(-\beta \Delta)_{\Lambda}^{-1}\left(\sqrt{f}-\sqrt{f_{\kappa}^{( \pm)}}\right)\right\| \\
+\left\|\sqrt{f_{\kappa}^{( \pm)}}\left[(-\beta \Delta)_{\Lambda}^{-1}-\kappa^{-2} K_{\Lambda}^{\beta / \kappa^{2}}\right] \sqrt{f_{\kappa}^{( \pm)}}\right\| \\
\left.\leqslant \quad\|\sqrt{f}\|_{\infty}+\left\|\sqrt{f_{\kappa}^{( \pm)}}\right\|_{\infty}\right)\left\|(-\beta \Delta)_{\Lambda}^{-1}\right\|\left\|\sqrt{f}-\sqrt{f_{\kappa}^{( \pm)}}\right\|_{\infty} \\
+\left\|\sqrt{f_{\kappa}^{( \pm)}}\right\|_{\infty}^{2}\left\|(-\beta \Delta)_{\Lambda}^{-1}-\kappa^{-2} K_{\Lambda}^{\beta / \kappa^{2}}\right\|=O\left(\kappa^{-2}\right) .
\end{gathered}
$$

Lemma 3.8 The operator $K_{\Lambda}^{\beta / \kappa^{2}} \in \mathfrak{C}_{1}\left(L^{2}\left(\mathbb{R}^{d}\right)\right)$, i.e. belongs to the trace-class operators on $L^{2}\left(\mathbb{R}^{d}\right)$, and

$$
\operatorname{Tr}\left[\sqrt{f} K_{\Lambda}^{\beta / \kappa^{2}} \sqrt{f}\right]=\kappa^{d} \rho_{c} \int_{\mathbb{R}^{d}} f(x) d x
$$

Proof: Let $\left\{\phi_{n}\right\}_{n}$ be a complete ortho-normal system (CONS) functions in $L^{2}\left(\mathbb{R}^{d}\right)$ and let $g(x)=e^{i p \cdot x} \chi_{\Lambda}(x)$. Then we have

$$
\sum_{n}\left|\widetilde{\chi_{\Lambda} \phi_{n}}(p)\right|^{2}=\sum_{n} \frac{\left|\left\langle g, \phi_{n}\right\rangle\right|^{2}}{(2 \pi)^{d}}=\frac{\|g\|_{2}^{2}}{(2 \pi)^{d}}=\frac{\left\|\chi_{\Lambda}\right\|_{2}^{2}}{(2 \pi)^{d}} .
$$

This yields

$$
\begin{gathered}
\sum_{n}\left\langle\phi_{n}, K_{\Lambda}^{\beta / \kappa^{2}} \phi_{n}\right\rangle=\sum_{n} \int_{\mathbb{R}^{d}} \frac{1}{e^{\beta|p|^{2} / \kappa^{2}}-1}\left|\widetilde{\chi_{\Lambda} \phi_{n}}(p)\right|^{2} d p \\
=\left\|\chi_{\Lambda}\right\|_{2}^{2} \int_{\mathbb{R}^{d}} \frac{1}{e^{\beta|p|^{2} / \kappa^{2}}-1} \frac{d p}{(2 \pi)^{d}}=\kappa^{d} \rho_{c}|\Lambda|<\infty
\end{gathered}
$$

Since $K_{\Lambda}^{\beta / \kappa^{2}} \geq 0$, it follows that $K_{\Lambda}^{\beta / \kappa^{2}} \in \mathfrak{C}_{1}\left(L^{2}\left(\mathbb{R}^{d}\right)\right)$. Similarly, we obtain the explicit value (3.1).

Lemma 3.9 The operator $K_{\Lambda}^{\beta / \kappa^{2}} \geq 0$ verifies the following Hilbert-Schmidt norm estimate from above:

$$
\left\|K_{\Lambda}^{\beta / \kappa^{2}}\right\|_{H S}^{2} \leqslant c_{d}\left(\kappa^{2} / \beta\right)^{(d \vee 4) / 2}\left(1+\left|\log \left(\kappa^{2} / \beta\right)\right|\right)|\Lambda|(1+|\Lambda|) .
$$

Here $c_{d}$ is a constant depending only on the dimension $d>2$.

Proof: By the Fourier transformation, we obtain

$$
\begin{aligned}
\left\|K_{\Lambda}^{\beta / \kappa^{2}}\right\|_{H S}^{2} & =\int_{\mathbb{R}^{d}} \frac{d q}{(2 \pi)^{d / 2}} \int_{\mathbb{R}^{d}} \frac{d p}{(2 \pi)^{d / 2}} \frac{\left|\widetilde{\chi_{\Lambda}}(p-q)\right|^{2}}{\left(e^{\beta|p|^{2} / \kappa^{2}}-1\right)\left(e^{\beta|q|^{2} / \kappa^{2}}-1\right)} \\
& =\int_{\mathbb{R}^{d}} \frac{d q}{(2 \pi)^{d / 2}} \int_{\mathbb{R}^{d}} \frac{d p}{(2 \pi)^{d / 2}} \frac{\left|\widetilde{\chi_{\Lambda}}(p)\right|^{2}}{\left(e^{\beta|p+q|^{2} / \kappa^{2}}-1\right)\left(e^{\beta|q|^{2} / \kappa^{2}}-1\right)} .
\end{aligned}
$$


$1^{\circ}$. Case : $2<d<4$.

From (3.4), we obtain the estimate:

$$
\begin{aligned}
\left\|K_{\Lambda}^{\beta / \kappa^{2}}\right\|_{H S}^{2} & \leqslant \int_{\mathbb{R}^{d}} \frac{d q}{(2 \pi)^{d / 2}} \int_{\mathbb{R}^{d}} \frac{d p}{(2 \pi)^{d / 2}} \frac{\kappa^{4}\left|\widetilde{\chi_{\Lambda}}(p)\right|^{2}}{\beta^{2}|p+q|^{2}|q|^{2}} \\
& =\left(\frac{\kappa^{2}}{\beta}\right)^{4 / 2} \int_{\mathbb{R}^{d}} \frac{\left|\widetilde{\chi_{\Lambda}}(p)\right|^{2}}{|p|^{4-d}} \frac{d p}{(2 \pi)^{d}} \int_{\mathbb{R}^{d}} \frac{d \tilde{q}}{|e+\tilde{q}|^{2}|\tilde{q}|^{2}} \\
& \leqslant\left(\frac{\kappa^{2}}{\beta}\right)^{4 / 2} c\left[\int_{|p| \leqslant 1} \frac{\left\|\widetilde{\chi_{\Lambda}}\right\|_{\infty}^{2}}{|p|^{4-d}} d p+\int_{|p|>1}\left|\widetilde{\chi_{\Lambda}}(p)\right|^{2} d p\right] \\
& \leqslant\left(\frac{\kappa^{2}}{\beta}\right)^{4 / 2} c\left(\left\|\chi_{\Lambda}\right\|_{1}^{2}+\left\|\chi_{\Lambda}\right\|_{2}^{2}\right)=\left(\frac{\kappa^{2}}{\beta^{2}}\right)^{4 / 2} c\left(|\Lambda|^{2}+|\Lambda|\right) .
\end{aligned}
$$

Here we changed the variable $q=|p| \tilde{q}$ in the first equality, and we denote by $e$ a unit vector in $\mathbb{R}^{d}$.

$2^{\circ}$. Case : $d>4$.

We apply the Cauchy-Schwarz inequality to (3.3) to get

$$
\begin{aligned}
\left\|K_{\Lambda}^{\beta / \kappa^{2}}\right\|_{H S}^{2} & \leqslant \sqrt{\iint \frac{\left|\widetilde{\chi_{\Lambda}}(p-q)\right|^{2} d p d q}{\left(e^{\beta|p|^{2} / \kappa^{2}}-1\right)^{2}(2 \pi)^{d}}} \sqrt{\iint \frac{\left|\widetilde{\chi_{\Lambda}}(p-q)\right|^{2} d p d q}{\left(e^{\beta|q|^{2} / \kappa^{2}}-1\right)^{2}(2 \pi)^{d}}} \\
& =\int_{\mathbb{R}^{d}}\left|\widetilde{\chi_{\Lambda}}(p)\right|^{2} \frac{d p}{(2 \pi)^{d}} \int_{\mathbb{R}^{d}} \frac{d q}{\left(e^{\beta|q|^{2} / \kappa^{2}}-1\right)^{2}} \\
& =c_{d}|\Lambda|\left(\frac{\kappa^{2}}{\beta}\right)^{d / 2} .
\end{aligned}
$$

$3^{\circ}$. Case : $d=4$.

Let us decompose (3.4) into two parts:

$$
\begin{aligned}
\left\|K_{\Lambda}^{\beta / \kappa^{2}}\right\|_{H S}^{2} & =\int_{\mathbb{R}^{d}} \frac{d p}{(2 \pi)^{d}}\left|\widetilde{\chi_{\Lambda}}(p)\right|^{2}\left\{\int_{|q| \geqslant 2|p|}+\int_{|q|<2|p|}\right\} \frac{d q}{\left(e^{\beta|p+q|^{2} / \kappa^{2}}-1\right)\left(e^{\beta|q|^{2} / \kappa^{2}}-1\right)} \\
& =\mathcal{I}_{1}+\mathcal{I}_{2} .
\end{aligned}
$$

For $\mathcal{I}_{1},|q| \geqslant 2|p|$ implies $|p+q| \geqslant|q|-|p| \geqslant|q| / 2$. Therefore, it follows that

$$
\begin{aligned}
\mathcal{I}_{1} \leqslant & \int_{\mathbb{R}^{d}} \frac{d p}{(2 \pi)^{d}}\left|\widetilde{\chi_{\Lambda}}(p)\right|^{2} \int_{|q| \geqslant 2|p|} \frac{d q}{\left(e^{\left.\beta|q|^{2} / 4 \kappa^{2}-1\right)\left(e^{\beta|q|^{2} / \kappa^{2}}-1\right)}\right.} \\
\leqslant & \int_{\mathbb{R}^{d}} \frac{d p}{(2 \pi)^{d}}\left|\widetilde{\chi_{\Lambda}}(p)\right|^{2}\left\{\theta(1-2|p|) \int_{1 \geqslant|q| \geqslant 2|p|}+\int_{\kappa / \sqrt{\beta} \geqslant|q|>1}+\int_{|q|>\kappa / \sqrt{\beta}}\right\} \\
& \times \frac{d q}{\left(e^{\beta|q|^{2} / 4 \kappa^{2}}-1\right)\left(e^{\beta|q|^{2} / \kappa^{2}}-1\right)}
\end{aligned}
$$




$$
\begin{aligned}
& \leqslant \int_{\mathbb{R}^{d}} \frac{d p}{(2 \pi)^{d}}\left|\widetilde{\chi_{\Lambda}}(p)\right|^{2}\left\{\theta(1-2|p|) \int_{1 \geqslant|q| \geqslant 2|p|} \frac{4 \kappa^{4} d q}{\beta^{2}|q|^{4}}+\int_{\kappa / \sqrt{\beta} \geqslant|q|>1} \frac{4 \kappa^{4} d q}{\beta^{2}|q|^{4}}\right. \\
& \left.+\int_{|q|>\kappa / \sqrt{\beta}} \frac{d q}{\left(e^{\beta|q|^{2} / 4 \kappa^{2}}-1\right)\left(e^{\beta|q|^{2} / \kappa^{2}}-1\right)}\right\} \\
& \leqslant \int_{\mathbb{R}^{d}} \frac{d p}{(2 \pi)^{d}}\left|\widetilde{\chi_{\Lambda}}(p)\right|^{2}\left\{\theta(1-2|p|) \frac{\kappa^{4}}{\beta^{2}} c_{1} \log \frac{1}{2|p|}+\frac{\kappa^{4}}{\beta^{2}} c_{2}\left|\log \left(\frac{\kappa^{2}}{\beta}\right)\right|\right. \\
& \left.+\left(\frac{\kappa^{2}}{\beta}\right)^{2} \int_{|\tilde{q}|>1} \frac{d \tilde{q}}{\left(e^{|\tilde{q}|^{2} / 4}-1\right)\left(e^{|\tilde{q}|^{2}}-1\right)}\right\} \\
& \leqslant\left\|\widetilde{\chi_{\Lambda}}\right\|_{\infty}^{2}\left(\frac{\kappa^{2}}{\beta}\right)^{4 / 2} c_{1} \int_{|p| \leqslant 1 / 2} \log \frac{1}{2|p|} d p+\left(\frac{\kappa^{2}}{\beta}\right)^{4 / 2}\left(c_{2}\left|\log \left(\frac{\kappa^{2}}{\beta}\right)\right|+c_{3}\right)\left\|\chi_{\Lambda}\right\|_{2}^{2} \\
& \leqslant c_{4}\left(\frac{\kappa^{2}}{\beta}\right)^{4 / 2}\left(1+\left|\log \left(\kappa^{2} / \beta\right)\right|\right)\left(|\Lambda|+|\Lambda|^{2}\right) \text {. }
\end{aligned}
$$

For $\mathcal{I}_{2}$, we obtain:

$$
\begin{aligned}
\mathcal{I}_{2} & \leqslant \int_{\mathbb{R}^{4}} \frac{d p}{(2 \pi)^{d}}\left|\widetilde{\chi_{\Lambda}}(p)\right|^{2} \int_{|q|<2|p|} \frac{\kappa^{4} d q}{\beta^{2}|p+q|^{2}|q|^{2}} \\
& =\int_{\mathbb{R}^{d}} \frac{d p}{(2 \pi)^{d}}\left|\widetilde{\chi_{\Lambda}}(p)\right|^{2} \int_{|\tilde{q}|<2} \frac{\kappa^{4} d \tilde{q}}{\beta^{2}|e+\tilde{q}|^{2}|\tilde{q}|^{2}} \\
& =c\left(\frac{\kappa^{2}}{\beta}\right)^{4 / 2}|\Lambda| .
\end{aligned}
$$

Thus, we have obtained the desired estimate (3.2) for all cases.

\section{Limit theorems for BEC}

In this section, we consider the boson random point processus (perfect Bose-gas) in the regime condensation, i.e. when

$$
\rho>\rho_{c}\left(=\rho_{c}(\beta)\right) \quad \text { and } \quad \nu_{\rho}=\mu_{K^{\beta}}^{(d e t)} * \mu_{K^{\beta},\left(\rho-\rho_{c}\right)},
$$

where $\rho_{c}(\beta)=K^{\beta}(x, x)=\int_{\mathbb{R}^{d}} \frac{1}{e^{\beta|p|^{2}}-1} \frac{d p}{(2 \pi)^{d}}$.

Proposition 4.1 For a bounded measurable set $\Lambda \subset \mathbb{R}^{d}$ and non-negative bounded function $f$ with supp $f \subset \Lambda$, one gets the equalities:

$$
\int_{Q\left(\mathbb{R}^{d}\right)}\langle f, \xi\rangle \nu_{\rho}(d \xi)=\rho \int_{\mathbb{R}^{d}} f(x) d x,
$$

and

$\int_{Q\left(\mathbb{R}^{d}\right)}\left(\langle f, \xi\rangle-\int_{Q\left(\mathbb{R}^{d}\right)}\langle f, \xi\rangle \nu_{\rho}(d \xi)\right)^{2} \nu_{\rho}(d \xi)=\rho \int_{\mathbb{R}^{d}} f(x)^{2} d x+\operatorname{Tr}\left[f K_{\Lambda}^{\beta} f K_{\Lambda}^{\beta}\right]+2\left(\rho-\rho_{c}\right)\left\langle f, K_{\Lambda}^{\beta} f\right\rangle$. 
Proof : Let us put

$$
e^{-W(f)}:=\int_{Q\left(\mathbb{R}^{d}\right)} e^{-\langle f, \xi\rangle} \nu_{\rho}(d \xi)
$$

then from (2.1) and (2.2) we get

$$
W(f)=\left(\rho-\rho_{c}\right)\left\langle\chi_{\Lambda},\left(1+\left(1-e^{-f}\right) K_{\Lambda}^{\beta}\right)^{-1}\left(1-e^{-f}\right)\right\rangle+\log \operatorname{Det}\left[1+\left(1-e^{-f}\right) K_{\Lambda}^{\beta}\right] .
$$

For small $\epsilon>0$, this yields the expansion:

$W(\epsilon f)=\epsilon \rho \int_{\mathbb{R}^{d}} f(x) d x-\frac{\epsilon^{2}}{2} \rho \int_{\mathbb{R}^{d}} f(x)^{2} d x-\frac{\epsilon^{2}}{2} \operatorname{Tr}\left[f K_{\Lambda}^{\beta} f K_{\Lambda}^{\beta}\right]-\epsilon^{2}\left(\rho-\rho_{c}\right)\left\langle f, K_{\Lambda}^{\beta} f\right\rangle+O\left(\epsilon^{3}\right)$,

which implies the proposition.

Corollary 4.2 Under the same conditions as in the Proposition 4.1, one obtains, for large $\kappa$, the following asymptotics :

$$
\int_{Q\left(\mathbb{R}^{d}\right)}\langle f(\dot{\bar{\kappa}}), \xi\rangle \nu_{\rho}(d \xi)=\kappa^{d} \rho \int_{\mathbb{R}^{d}} f(x) d x+o\left(\kappa^{d}\right),
$$

and

$$
\begin{aligned}
& \int_{Q\left(\mathbb{R}^{d}\right)}\left(\langle f(\dot{\dot{\kappa}}), \xi\rangle-\int_{Q\left(\mathbb{R}^{d}\right)}\langle f(\dot{\bar{\kappa}}), \xi\rangle \nu_{\rho}(d \xi)\right)^{2} \nu_{\rho}(d \xi) \\
& =2 \kappa^{d+2}\left(\rho-\rho_{c}\right)\left\langle f,(-\beta \Delta)_{\Lambda}^{-1} f\right\rangle+O\left(\kappa^{4 \vee d} \log \kappa\right) .
\end{aligned}
$$

Proof: Using the unitary operator $U_{\kappa}$, we get

$$
\begin{gathered}
\operatorname{Tr}\left[f(\cdot / \kappa) K_{\kappa \Lambda}^{\beta} f(\cdot / \kappa) K_{\kappa \Lambda}^{\beta}\right]=\operatorname{Tr}\left[U_{\kappa} f(\cdot / \kappa) K_{\kappa \Lambda}^{\beta} f(\cdot / \kappa) K_{\kappa \Lambda}^{\beta} U_{\kappa}^{-1}\right] \\
=\operatorname{Tr}\left[f K_{\Lambda}^{\beta / \kappa^{2}} f K_{\Lambda}^{\beta / \kappa^{2}}\right] \leqslant\|f\|_{\infty}^{2}\left\|K_{\Lambda}^{\beta / \kappa^{2}}\right\|_{H S}^{2}=O\left(\kappa^{d \vee 4} \log \kappa\right)
\end{gathered}
$$

and

$$
\begin{aligned}
& \left\langle f(\cdot / \kappa), K_{\kappa \Lambda}^{\beta} f(\cdot / \kappa)\right\rangle=\left\langle U_{\kappa} f(\cdot / \kappa), U_{\kappa} K_{\kappa \Lambda}^{\beta} f(\cdot / \kappa)\right\rangle \\
& =\kappa^{d}\left\langle f, K_{\Lambda}^{\beta \kappa^{-2}} f\right\rangle=\kappa^{d+2}\left\langle f,(-\beta \Delta)_{\Lambda}^{-1} f\right\rangle+O\left(\kappa^{d}\right) .
\end{aligned}
$$

Here we used Lemma 3.9 and Lemma 3.6. Note that $\operatorname{supp} f(\cdot / \kappa) \subset \kappa \Lambda$. Then Proposition 4.1 yields the Corollary.

Theorem 4.3 (The law of large number) For $\kappa \rightarrow \infty$ and for any bounded function $f$ with compact support the limit

$$
\frac{1}{\kappa^{d}}\left\langle f\left(\frac{\dot{\kappa}}{\kappa}\right), \xi\right\rangle \longrightarrow \rho \int_{\mathbb{R}^{d}} f(x) d x
$$

holds in $L^{2}\left(Q\left(\mathbb{R}^{d}\right), \nu_{\rho}\right)$. 
Proof: This is a simple consequence of the Corollary 4.2 .

Theorem 4.4 (Central Limit Theorem) For $\kappa \rightarrow \infty$ the family of random variables

$$
X_{\kappa}=\kappa^{-(d+2) / 2} \frac{\langle f(\cdot / \kappa), \xi\rangle-\rho \kappa^{d} \int_{\mathbb{R}^{d}} f(x) d x}{\sqrt{2\left(\rho-\rho_{c}\right)\left\langle f,(-\beta \Delta)_{\Lambda}^{-1} f\right\rangle}}
$$

converges in distribution to the standard Gaussian random variable.

Proof: By Theorem 2.1(1),(3), we obtain

$$
\begin{gathered}
\mathbb{E}_{\nu_{\rho}}\left[\exp \left[i \lambda \kappa^{-(d+2) / 2}\left(\langle f(\cdot / \kappa), \xi\rangle-\rho \kappa^{d} \int_{\mathbb{R}^{d}} f(x) d x\right)\right]\right] \\
=\exp \left[-i \lambda \kappa^{(d-2) / 2} \rho \int_{\mathbb{R}^{d}} f(x) d x-W_{\kappa}\right]
\end{gathered}
$$

where

$$
\begin{gathered}
W_{\kappa}=\left(\rho-\rho_{c}\right)\left\langle\chi_{\kappa \Lambda},\left(1+\left(1-e^{i \lambda \kappa^{-(d+2) / 2} f(\cdot / \kappa)}\right) K_{\kappa \Lambda}^{\beta}\right)^{-1}\left(1-e^{i \lambda \kappa^{-(d+2) / 2} f(\cdot / \kappa)}\right)\right\rangle \\
+\log \operatorname{Det}\left[1+\left(1-e^{i \lambda \kappa^{-(d+2) / 2} f(\cdot / \kappa)}\right) K_{\kappa \Lambda}^{\beta}\right] .
\end{gathered}
$$

By definition of transformation $U_{\kappa}$ and by Lemma 3.6, the first term can be expanded as

$$
\begin{gathered}
\left(\rho-\rho_{c}\right)\left\langle U_{\kappa} \chi_{\kappa \Lambda}, U_{\kappa}\left(1+\left(1-e^{i \lambda \kappa^{-(d+2) / 2} f(\cdot / \kappa)}\right) K_{\kappa \Lambda}^{\beta}\right)^{-1}\left(1-e^{i \lambda \kappa^{-(d+2) / 2} f(\cdot / \kappa)}\right)\right\rangle \\
\quad=-i \lambda\left(\rho-\rho_{c}\right) \kappa^{(d-2) / 2}\left[\int f d x+i \lambda \kappa^{-(d+2) / 2}\left\langle f, K_{\Lambda}^{\beta / \kappa^{2}} f\right\rangle\right]+o(1) \\
=-i \lambda\left(\rho-\rho_{c}\right) \kappa^{(d-2) / 2} \int f d x+\lambda^{2}\left(\rho-\rho_{c}\right)\left\langle f,(-\beta \Delta)_{\Lambda}^{-1} f\right\rangle+o(1) .
\end{gathered}
$$

Here we applied the bound:

$$
\left\|(1-Y)^{-1}-(1+Y)\right\| \leqslant c\|Y\|^{2}
$$

valid for operators with small enough operator norms with a bound defined by $c$.

Similarly, we get also the representation for the second term:

$$
\begin{gathered}
\log \operatorname{Det}\left[1+\left(1-e^{i \lambda \kappa^{-(d+2) / 2} f}\right) K_{\Lambda}^{\beta / \kappa^{2}}\right] \\
=-i \lambda \kappa^{-(d+2) / 2} \operatorname{Tr}\left[f K_{\Lambda}^{\beta / \kappa^{2}}\right]+R,
\end{gathered}
$$

where

$$
\operatorname{Tr}\left[f K_{\Lambda}^{\beta / \kappa^{2}}\right]=\rho_{c} \kappa^{d} \int f(x) d x
$$

and

$$
|R| \leqslant \operatorname{Tr}\left[\left(\left(1-e^{i \lambda \kappa^{-(d+2) / 2} f}\right) K_{\Lambda}^{\beta / \kappa^{2}}\right)^{2}\right]
$$




$$
=O\left(\lambda^{2} \kappa^{-d-2}\right)\|f\|_{\infty}^{2}\left\|K_{\Lambda}^{\beta / \kappa^{2}}\right\|_{H S}^{2}=o(1) .
$$

Here we used the bound:

$$
|\log \operatorname{Det}[1+Y]-\operatorname{Tr} Y|=\left|\log \operatorname{Det}_{2}[1+Y]\right|=O\left(\|Y\|_{H S}^{2}\right)
$$

for the trace-class operators with small operator norms. Recall that $\operatorname{Det}_{2}[1+Y]:=$ $e^{-\operatorname{Tr} Y} \operatorname{Det}[1+Y]=\operatorname{Det}\left[(1-Y) e^{-Y}\right]$ denotes a "regularized" determinant for the HilbertSchmidt operators $Y$, see e.g. ShTa].

Thus we get

$$
W_{\kappa}=-i \lambda \rho \kappa^{(d-2) / 2} \int f d x+\lambda^{2}\left(\rho-\rho_{c}\right)\left\langle f,(-\beta \Delta)_{\Lambda}^{-1} f\right\rangle+o(1)
$$

and

$$
\begin{gathered}
\mathbb{E}_{\nu_{\rho}}\left[\exp \left[i \lambda \kappa^{-(d+2) / 2}\left(\langle f(\cdot / \kappa), \xi\rangle-\rho \kappa^{d} \int_{\mathbb{R}^{d}} f(x) d x\right)\right]\right] \\
=e^{-\lambda^{2}\left(\rho-\rho_{c}\right)\left\langle f,(-\beta \Delta)_{\Lambda}^{-1} f\right\rangle+o(1)} .
\end{gathered}
$$

Then setting $\lambda:=t / \sqrt{2\left(\rho-\rho_{c}\right)\left\langle f,(-\beta \Delta)_{\Lambda}^{-1} f\right\rangle}$, we finally obtain the limit:

$$
\mathbb{E}_{\nu_{\rho}}\left[e^{i t X_{\kappa}}\right] \rightarrow e^{-t^{2} / 2}
$$

which finishes the proof of the Central Limit Theorem.

Remark 4.5 The above calculations show that the value of the variation that we need to normalize the limiting random variable, is contributed from the measure $\mu_{K^{\beta},\left(\rho-\rho_{c}\right)}$.

Before to pass to the Large Deviation Principle, we prove the following lemma.

Lemma 4.6 Let $\left\|\sqrt{f}(-\beta \Delta)_{\Lambda}^{-1} \sqrt{f}\right\|<1$. Then $-\beta \Delta-f$ is a self-adjoint operator, which satisfies the property : Spec $(-\beta \Delta-f) \subset[0, \infty)$. Moreover, the operator $(-\beta \Delta-f)_{\Lambda}^{-1}$ is bounded and we have:

$$
\left\langle\sqrt{f},\left[1-\sqrt{f}(-\beta \Delta)_{\Lambda}^{-1} \sqrt{f}\right]^{-1} \sqrt{f}\right\rangle=\int_{\mathbb{R}^{d}} f(x) d x+\left\langle f,(-\beta \Delta-f)_{\Lambda}^{-1} f\right\rangle .
$$

Proof: Since the operator $-\beta \Delta$ is self-adjoint, the spectrum $\operatorname{Spec}(-\beta \Delta) \subset[0, \infty)$ and $f$ is a bounded function, it is obvious that $-\beta \Delta-f$ is self-adjoint and $(\delta-\beta \Delta)^{-1}$ is bounded non-negative operator for arbitrary $\delta>0$. Since $f \geqslant 0$ and $\operatorname{supp} f \subset \Lambda$, it is also obvious that

$$
0 \leqslant \sqrt{f}(\delta-\beta \Delta)^{-1} \sqrt{f} \leqslant \sqrt{f}(-\beta \Delta)_{\Lambda}^{-1} \sqrt{f} .
$$

Together with the assumption $\left\|\sqrt{f}(-\beta \Delta)_{\Lambda}^{-1} \sqrt{f}\right\|<1$, the operator

$$
S:=(\delta-\beta \Delta)^{-1}+(\delta-\beta \Delta)^{-1} \sqrt{f} \sum_{n=0}^{\infty}\left(\sqrt{f}(\delta-\beta \Delta)^{-1} \sqrt{f}\right)^{n} \sqrt{f}(\delta-\beta \Delta)^{-1}
$$


is a bounded non-negative operator. On the other hand, one can check that

$$
(\delta-\beta \Delta-f) S=I \quad \text { and } \quad S(\delta-\beta \Delta-f)=I_{\operatorname{Dom}(\Delta)},
$$

which implies that $S=(\delta-\beta \Delta-f)^{-1}$. Thus, we have $-\delta \notin \operatorname{Spec}(-\beta \Delta-f)$, i.e., Spec $-\beta \Delta-f \subset[0, \infty)$. Let $\{E(\lambda)\}$ be the spectral decomposition of the operator $(-\beta \Delta-f)$. Then $E(-0)=0$. Moreover, $E(0)=0$ holds. Indeed, if one supposes the contrary, then there exists a $\psi \neq 0$ such that

$$
\psi \in E(0) L^{2}\left(\mathbb{R}^{d}\right) \quad \text { and } \quad(-\beta \Delta-f) \psi=0 \text {. }
$$

Thus, we have $f \psi=-\beta \Delta \psi$, which implies that

$$
f \psi \in \operatorname{Ran}(-\beta \Delta)=\operatorname{Dom}(-\beta \Delta)^{-1}
$$

and

$$
\psi=(-\beta \Delta)^{-1} f \psi
$$

Hence we get $\sqrt{f} \psi=\sqrt{f}(-\beta \Delta)^{-1} f \psi=\left(\sqrt{f}(-\beta \Delta)_{\Lambda}^{-1} \sqrt{f}\right) \sqrt{f} \psi$. This contradicts the estimate $\left\|\sqrt{f}(-\beta \Delta)_{\Lambda}^{-1} \sqrt{f}\right\|<1$ because $\sqrt{f} \psi \in L^{2}\left(\mathbb{R}^{d}\right)$ belong to the eigenvalue 1 of the operator $\sqrt{f}(-\beta \Delta)_{\Lambda}^{-1} \sqrt{f}$. Therefore, we obtain densely defined non-negative selfadjoint operator:

$$
(-\beta \Delta-f)^{-1}:=\int_{0}^{\infty} \frac{d E(\lambda)}{\lambda} .
$$

The boundedness of $(-\beta \Delta-f)_{\Lambda}^{-1}$ follows from the estimates:

$$
\begin{aligned}
\left\|(-\beta \Delta-f)_{\Lambda}^{-1}\right\| & =\sup _{\|\phi\|_{2}=1} \int_{0}^{\infty} \frac{d\left\langle\chi_{\Lambda} \phi, E(\lambda) \chi_{\Lambda} \phi\right\rangle}{\lambda} \\
& =\sup _{\|\phi\|_{2}=1} \lim _{\delta \downarrow 0} \int_{0}^{\infty} \frac{d\left\langle\chi_{\Lambda} \phi, E(\lambda) \chi_{\Lambda} \phi\right\rangle}{\delta+\lambda}=\sup _{\|\phi\|_{2}=1} \lim _{\delta \downarrow 0}\left\langle\chi_{\Lambda} \phi, S \chi_{\Lambda} \phi\right\rangle \\
& \leqslant \sup _{\|\phi\|_{2}=1}\left[\left\langle\phi,(-\beta \Delta)_{\Lambda}^{-1} \phi\right\rangle+\frac{\left\|\sqrt{f}(\delta-\beta \Delta)^{-1} \chi_{\Lambda} \phi\right\|_{2}^{2}}{1-\left\|\sqrt{f}(-\beta \Delta)_{\Lambda}^{-1} \sqrt{f}\right\|}\right] \\
& \leqslant\left\|(-\beta \Delta)_{\Lambda}^{-1}\right\|+\frac{\|f\|_{\infty}\left\|(\delta-\beta \Delta)_{\Lambda}^{-1}\right\|^{2}}{1-\left\|\sqrt{f}(-\beta \Delta)_{\Lambda}^{-1} \sqrt{f}\right\|}<\infty .
\end{aligned}
$$

To derive equation (4.2), we exploit the operator (4.3) for $\delta \downarrow 0$ :

$$
\begin{gathered}
\left\langle f,(\delta-\beta \Delta-f)^{-1} f\right\rangle=\left\langle\sqrt{f}, \sum_{n=1}^{\infty}\left(\sqrt{f}(\delta-\beta \Delta)^{-1} \sqrt{f}\right)^{n} \sqrt{f}\right\rangle \\
=-\langle\sqrt{f}, \sqrt{f}\rangle+\left\langle\sqrt{f},\left(1-\sqrt{f}(\delta-\beta \Delta)^{-1} \sqrt{f}\right)^{-1} \sqrt{f}\right\rangle \\
\longrightarrow-\int f d x+\left\langle\sqrt{f},\left(1-\sqrt{f}(-\beta \Delta)_{\Lambda}^{-1} \sqrt{f}\right)^{-1} \sqrt{f}\right\rangle
\end{gathered}
$$


where we used the convergence

$$
\sqrt{f}(\delta-\beta \Delta)^{-1} \sqrt{f} \rightarrow \sqrt{f}(-\beta \Delta)_{\Lambda}^{-1} \sqrt{f}
$$

in the operator norm. The latter is a direct consequence of the spectral theorem and the dominated convergence theorem. On the other hand, we notice that for $\delta \downarrow 0$ one gets by the monotone convergence theorem the limit:

$$
\begin{gathered}
\left\langle f,(\delta-\beta \Delta-f)^{-1} f\right\rangle=\int_{0}^{\infty} \frac{d\left\langle\chi_{\Lambda} f, E(\lambda) \chi_{\Lambda} f\right\rangle}{\delta+\lambda} \\
\longrightarrow \int_{0}^{\infty} \frac{d\left\langle\chi_{\Lambda} f, E(\lambda) \chi_{\Lambda} f\right\rangle}{\lambda}=\left\langle f,(-\beta \Delta-f)_{\Lambda}^{-1} f\right\rangle .
\end{gathered}
$$

Therefore, the equality (4.2) is proven.

Theorem 4.7 For any bounded measurable function $f \geqslant 0$ with bounded support and for any bounded measurable subset $\Lambda$ of $\mathbb{R}^{d}$ satisfying $\operatorname{supp} f \subset \Lambda$ we have the following limits:

$$
\begin{gathered}
P(t):=\lim _{\kappa \rightarrow \infty} \frac{1}{\kappa^{d-2}} \log \int_{Q\left(\mathbb{R}^{d}\right)} e^{t \kappa^{-2}\langle f(\cdot / \kappa), \xi\rangle} \nu_{\rho}(d \xi) \\
= \begin{cases}\rho t \int_{\mathbb{R}^{d}} f(x) d x+\left(\rho-\rho_{c}\right) t^{2}\left\langle f,(-\beta \Delta-t f)_{\Lambda}^{-1} f\right\rangle & \text { for } t \in\left(-\infty,\left\|\sqrt{f}(-\beta \Delta)_{\Lambda}^{-1} \sqrt{f}\right\|^{-1}\right), \\
\infty & \text { for } t \in\left[\left\|\sqrt{f}(-\beta \Delta)_{\Lambda}^{-1} \sqrt{f}\right\|^{-1}, \infty\right) .\end{cases}
\end{gathered}
$$

Remark 4.8 (1) If $t<\left\|\sqrt{f}(\beta \Delta)_{\Lambda}^{-1} \sqrt{f}\right\|^{-1}$, then from Lemma 4.0 we obtain the expression for the function $P(t)$ :

$$
P(t)=\rho_{c} t \int_{\mathbb{R}^{d}} f(x) d x+\left(\rho-\rho_{c}\right) t\left\langle\sqrt{f},\left[1-t \sqrt{f}(-\beta \Delta)_{\Lambda}^{-1} \sqrt{f}\right]^{-1} \sqrt{f}\right\rangle .
$$

(2) By Lemmas 3.9 and 3.7 the operator $\sqrt{f}(-\beta \Delta)_{\Lambda}^{-1} \sqrt{f}$ is a non-negative compact operator. Let $\left\{\varphi_{n}\right\}$ be a CONS of $L^{2}\left(\mathbb{R}^{2}\right)$, which consists of the eigenfunctions of this operator. We order the corresponding eigenvalues as

$$
\lambda_{1} \geqslant \lambda_{2} \geqslant \cdots \geqslant \lambda_{n} \geqslant \cdots \geqslant 0
$$

Then the Perron-Frobenius theorem yields

$$
\lambda_{1}=\left\|\sqrt{f}(-\beta \Delta)_{\Lambda}^{-1} \sqrt{f}\right\| \quad \text { and } \quad\left\langle\sqrt{f}, \varphi_{1}\right\rangle>0 .
$$

By the remark (1), we obtain:

$$
P(t)=\rho_{c} t \int_{\mathbb{R}^{d}} f(x) d x+\left(\rho-\rho_{c}\right) t \sum_{n=1}^{\infty} \frac{\left|\left\langle\sqrt{f}, \varphi_{n}\right\rangle\right|^{2}}{1-t \lambda_{n}} \quad \text { for } \quad t<\lambda_{1}^{-1},
$$


which ensures the (essential) smoothness of $P$ :

$$
P \text { is a } C^{\infty} \text { function on }\left(-\infty, \lambda_{1}^{-1}\right) \text { and } \lim _{t \uparrow \lambda_{1}^{-1}} P(t)=\infty \text {. }
$$

(3) Below we prove that the limits of the function $P$ for the components $\mu_{K, \rho}$ and $\mu_{K}^{(d e t)}$ of the boson random point processes have the following forms:

$$
\begin{gathered}
P_{K^{\beta}, \rho}(t):=\lim _{\kappa \rightarrow \infty} \frac{1}{\kappa^{d-2}} \log \int_{Q\left(\mathbb{R}^{d}\right)} e^{t \kappa^{-2}\langle f(\cdot / \kappa), \xi\rangle} \mu_{K^{\beta}, \rho}(d \xi) \\
= \begin{cases}\rho t\left\langle\sqrt{f},\left[1-t \sqrt{f}(-\beta \Delta)_{\Lambda}^{-1} \sqrt{f}\right]^{-1} \sqrt{f}\right\rangle & \text { for } t \in\left(-\infty,\left\|\sqrt{f}(-\beta \Delta)_{\Lambda}^{-1} \sqrt{f}\right\|^{-1}\right), \\
\infty & \text { for } t \in\left[\left\|\sqrt{f}(-\beta \Delta)_{\Lambda}^{-1} \sqrt{f}\right\|^{-1}, \infty\right),\end{cases}
\end{gathered}
$$

and

$$
\begin{gathered}
P_{K^{\beta}}^{(d e t)}(t):=\lim _{\kappa \rightarrow \infty} \frac{1}{\kappa^{d-2}} \log \int_{Q\left(\mathbb{R}^{d}\right)} e^{t \kappa^{-2}\langle f(\cdot / \kappa), \xi\rangle} \mu_{K^{\beta}}^{(d e t)}(d \xi) \\
= \begin{cases}\rho_{c} t \int_{\mathbb{R}^{d}} f(x) d x & \text { for } t \in\left(-\infty,\left\|\sqrt{f}(-\beta \Delta)_{\Lambda}^{-1} \sqrt{f}\right\|^{-1}\right), \\
\infty & \text { for } t \in\left[\left\|\sqrt{f}(-\beta \Delta)_{\Lambda}^{-1} \sqrt{f}\right\|^{-1}, \infty\right) .\end{cases}
\end{gathered}
$$

Proof (of Theorem 4.7): The proof consists of two parts corresponding to $t<0$ and $t>0$. ( The case $t=0$ is obvious.)

$1^{\circ}$. For $t<0$, it is enough to show that

$$
\begin{aligned}
& P(-1)=\lim _{\kappa \rightarrow \infty} \frac{1}{\kappa^{d-2}} \log \int_{Q\left(\mathbb{R}^{d}\right)} e^{-\kappa^{-2}\langle f(\cdot / \kappa), \xi\rangle} \nu_{\rho}(d \xi) \\
& =-\rho \int_{\mathbb{R}^{d}} f(x) d x+\left(\rho-\rho_{c}\right)\left\langle f,(-\beta \Delta+f)_{\Lambda}^{-1} f\right\rangle .
\end{aligned}
$$

To this end notice that from (2.1) and (2.2), together with the unitary transformation $U_{\kappa}$, one obtains the representation:

$$
\begin{gathered}
\frac{1}{\kappa^{d-2}} \log \int_{Q\left(\mathbb{R}^{d}\right)} e^{-\kappa^{-2}\langle f(\cdot / \kappa), \xi\rangle} \nu_{\rho}(d \xi) \\
=-\frac{\rho-\rho_{c}}{\kappa^{d-2}}\left\langle U_{\kappa} \sqrt{1-e^{-\kappa^{-2} f(\cdot / \kappa)}}, U_{\kappa}\left(1+\sqrt{1-e^{-\kappa^{-2} f(\cdot / \kappa)}} K_{\kappa \Lambda}^{\beta} \sqrt{\left.1-e^{-\kappa^{-2} f(\cdot / \kappa)}\right)^{-1} \sqrt{\left.1-e^{-\kappa^{-2} f(\cdot / \kappa)}\right\rangle}}\right.\right. \\
-\frac{1}{\kappa^{d-2}} \log \operatorname{Det}\left[1+U_{\kappa}\left(1-e^{-\kappa^{-2} f(\cdot / \kappa)}\right) K_{\kappa \Lambda}^{\beta} U_{\kappa}^{-1}\right] \\
=-\left(\rho-\rho_{c}\right)\left\langle\sqrt{f_{\kappa}^{(-)}},\left(1+\sqrt{f_{\kappa}^{(-)}} \kappa^{-2} K_{\Lambda}^{\beta / \kappa^{2}} \sqrt{f_{\kappa}^{(-)}}\right)^{-1} \sqrt{f_{\kappa}^{(-)}}\right\rangle \\
-\frac{1}{\kappa^{d}} \operatorname{Tr}\left[\sqrt{f_{\kappa}^{(-)}} K_{\Lambda}^{\beta / \kappa^{2}} \sqrt{f_{\kappa}^{(-)}}\right]-\frac{1}{\kappa^{d-2}} \log \operatorname{Det}_{2}\left[1+f_{\kappa}^{(-)} \kappa^{-2} K_{\Lambda}^{\beta / \kappa^{2}}\right],
\end{gathered}
$$


see Definition 3.4. Then we apply Lemmas 3.5, 3.7 to the first term, Lemma 3.8 to the second term and Lemma 3.9 with (4.1) to the third term to obtain:

$$
P(-1)=-\left(\rho-\rho_{c}\right)\left\langle\sqrt{f},\left[1+\sqrt{f}(-\beta \Delta)_{\Lambda}^{-1} \sqrt{f}\right]^{-1} \sqrt{f}\right\rangle-\rho_{c} \int_{\mathbb{R}^{d}} f(x) d x .
$$

Now it is sufficient to check the identity:

$$
\left\langle\sqrt{f},\left[1+\sqrt{f}(-\beta \Delta)_{\Lambda}^{-1} \sqrt{f}\right]^{-1} \sqrt{f}\right\rangle=\int_{\mathbb{R}^{d}} f(x) d x-\left\langle f,(-\beta \Delta+f)_{\Lambda}^{-1} f\right\rangle .
$$

Note that the inequality

$$
(-\beta \Delta+f)_{\Lambda}^{-1} \leqslant(-\beta \Delta)_{\Lambda}^{-1}
$$

yields that $(-\beta \Delta+f)_{\Lambda}^{-1}$ is bounded. Since the operators $(\epsilon-\beta \Delta)^{-1},(\epsilon-\beta \Delta+f)^{-1}$ are bounded and non-negative for any $\epsilon>0$, we get

$\sqrt{f}(\epsilon-\beta \Delta)^{-1} \sqrt{f}-\sqrt{f}(\epsilon-\beta \Delta+f)^{-1} \sqrt{f}=\sqrt{f}(\epsilon-\beta \Delta)^{-1} \sqrt{f} \sqrt{f}(\epsilon-\beta \Delta+f)^{-1} \sqrt{f}$.

It gives

$$
\sqrt{f}(\epsilon-\beta \Delta)^{-1} \sqrt{f}=\left(1+\sqrt{f}(\epsilon-\beta \Delta)^{-1} \sqrt{f}\right) \sqrt{f}(\epsilon-\beta \Delta+f)^{-1} \sqrt{f},
$$

which implies

$$
1-\left(1+\sqrt{f}(\epsilon-\beta \Delta)^{-1} \sqrt{f}\right)^{-1}=\sqrt{f}(\epsilon-\beta \Delta+f)^{-1} \sqrt{f} .
$$

Hence, to verify (4.5), it is enough to prove that

$$
\begin{array}{rlr}
\sqrt{f}(\epsilon-\beta \Delta+f)^{-1} \sqrt{f} & \rightarrow \sqrt{f}(-\beta \Delta+f)_{\Lambda}^{-1} \sqrt{f} & \text { weakly } \\
\sqrt{f}(\epsilon-\beta \Delta)^{-1} \sqrt{f} & \rightarrow \sqrt{f}(-\beta \Delta)_{\Lambda}^{-1} \sqrt{f} \quad \text { in norm } .
\end{array}
$$

To show (4.6), let $\{E(\lambda)\}$ be the spectral decomposition of $-\beta \Delta+f$. Since

$$
\begin{gathered}
\int_{0}^{\infty} \frac{d\langle\sqrt{f} \phi, E(\lambda) \sqrt{f} \phi\rangle}{\lambda}=\left\langle\phi, \sqrt{f}(-\beta \Delta+f)_{\Lambda}^{-1} \sqrt{f} \phi\right\rangle \\
\leqslant\left\langle\phi, \sqrt{f}(-\beta \Delta)_{\Lambda}^{-1} \sqrt{f} \phi\right\rangle<\infty
\end{gathered}
$$

holds for $\phi \in L^{2}\left(\mathbb{R}^{d}\right)$, the dominated convergence theorem yields the limit:

$$
\begin{gathered}
\left|\left\langle\phi, \sqrt{f}(-\beta \Delta+f)_{\Lambda}^{-1} \sqrt{f} \phi\right\rangle-\left\langle\phi, \sqrt{f}(\epsilon-\beta \Delta+f)_{\Lambda}^{-1} \sqrt{f} \phi\right\rangle\right| \\
=\int_{0}^{\infty}\left(\frac{1}{\lambda}-\frac{1}{\lambda+\epsilon}\right) d\langle\sqrt{f} \phi, E(\lambda) \sqrt{f} \phi\rangle \rightarrow 0 .
\end{gathered}
$$

To show (4.7), we use the Fourier transformation. Put

$$
\left\|\sqrt{f}(-\beta \Delta)_{\Lambda}^{-1} \sqrt{f}-\sqrt{f}(\epsilon-\beta \Delta)_{\Lambda}^{-1} \sqrt{f}\right\|
$$




$$
=\sup _{\|\phi\|_{2}=1} \int_{\mathbb{R}^{d}} \frac{\epsilon|\widetilde{\sqrt{f}} \phi(p)|^{2}}{\beta|p|^{2}\left(\epsilon+\beta|p|^{2}\right)} d p=: D .
$$

When $d>4$, we obtain that

$$
\begin{aligned}
D & \leqslant \sup _{\|\phi\|_{2}=1} \int_{|p|<1} \frac{\epsilon\|\widetilde{\sqrt{f} \phi}\|_{\infty}^{2}}{\beta^{2}|p|^{4}} d p+\sup _{\|\phi\|_{2}=1} \int_{|p| \geqslant 1} \frac{\epsilon|\widetilde{\sqrt{f} \phi}(p)|^{2}}{\beta^{2}} d p \\
& \leqslant \frac{\epsilon}{\beta^{2}}\left(c_{d}\|f\|_{1}+\|f\|_{\infty}\right) \rightarrow 0,
\end{aligned}
$$

for $\epsilon \rightarrow 0$. When $2<d<4$, we get

$$
\begin{gathered}
D \leqslant \sup _{\|\phi\|_{2}=1} \int_{\mathbb{R}^{d}} \frac{\epsilon\|\widetilde{\sqrt{f} \phi}\|_{\infty}^{2}}{\beta|p|^{2}\left(\epsilon+\beta|p|^{2}\right)} d p \\
\leqslant \frac{\epsilon^{(d-2) / 2}}{\beta^{d / 2}} \int_{\mathbb{R}^{d}} \frac{\|f\|_{1} d \tilde{p}}{(2 \pi)^{d}|\tilde{p}|^{2}\left(1+|\tilde{p}|^{2}\right)} \rightarrow 0,
\end{gathered}
$$

as $\epsilon \rightarrow 0$. Here we used the bounds $\|\widetilde{\sqrt{f} \phi}\|_{\infty} \leqslant(2 \pi)^{-d / 2}\|f\|_{1}^{1 / 2}\|\phi\|_{2}$ and $\|\widetilde{\sqrt{f} \phi}\|_{2} \leqslant$ $\|f\|_{\infty}^{1 / 2}\|\phi\|_{2}$, and changed the integral variable $p=\sqrt{\epsilon / \beta} \tilde{p}$ in the latter integral.

Similarly for $d=4$, we obtain the limit:

$$
\begin{aligned}
D & \leqslant \sup _{\|\phi\|_{2}=1} \int_{|p|<1} \frac{\epsilon\|\widetilde{\sqrt{f} \phi}\|_{\infty}^{2}}{\beta|p|^{2}\left(\epsilon+\beta|p|^{2}\right)} d p+\sup _{\|\phi\|_{2}=1} \int_{|p| \geqslant 1} \frac{\epsilon|\widetilde{\sqrt{f}} \phi(p)|^{2}}{\beta^{2}} d p \\
& \leqslant \frac{\epsilon^{(d-2) / 2}}{\beta^{d / 2}} \int_{|\tilde{p}|<\sqrt{\beta / \epsilon}} \frac{\|f\|_{1} d \tilde{p}}{(2 \pi)^{d}|\tilde{p}|^{2}\left(1+|\tilde{p}|^{2}\right)}+\frac{\epsilon}{\beta^{2}}\|f\|_{\infty} \\
& \leqslant c\|f\|_{1} \frac{\epsilon}{\beta^{2}} \log \left(1+\frac{\beta}{\epsilon}\right)+\frac{\epsilon}{\beta^{2}}\|f\|_{\infty} \rightarrow 0
\end{aligned}
$$

when $\epsilon \rightarrow 0$.

$2^{\circ}$. For $t>0$, It is enough to show

$$
P(1)= \begin{cases}\rho \int_{\mathbb{R}^{d}} f(x) d x+\left(\rho-\rho_{c}\right)\left\langle f,(-\beta \Delta-f)_{\Lambda}^{-1} f\right\rangle & \text { for }\left\|\sqrt{f}(-\beta \Delta)_{\Lambda}^{-1} \sqrt{f}\right\|<1, \\ \infty & \text { for }\left\|\sqrt{f}(-\beta \Delta)_{\Lambda}^{-1} \sqrt{f}\right\| \geqslant 1 .\end{cases}
$$

When $\left\|\sqrt{f}(-\beta \Delta)_{\Lambda}^{-1} \sqrt{f}\right\|<1$, then by Lemma 3.7 and Lemma 3.3 we have

$$
\left\|\sqrt{f_{\kappa}^{(+)}} \kappa^{-2} K_{\Lambda}^{\beta / \kappa^{2}} \sqrt{f_{\kappa}^{(+)}}\right\|=\left\|\sqrt{e^{\kappa^{-2} f(\cdot / \kappa)}-1} K_{\kappa \Lambda}^{\beta} \sqrt{e^{\kappa^{-2} f(\cdot / \kappa)}-1}\right\|<1
$$

for $\kappa$ large enough, see Definition 3.4. We also use Lemma 3.3 and Theorem 2.1 (2),(4) to obtain the representation:

$$
\frac{1}{\kappa^{d-2}} \log \int_{Q\left(\mathbb{R}^{d}\right)} e^{\kappa^{-2}\langle f(\cdot / \kappa), \xi\rangle} \nu_{\rho}(d \xi)
$$




$$
\begin{gathered}
=\frac{\rho-\rho_{c}}{\kappa^{d-2}}\left\langle U_{\kappa} \sqrt{e^{\kappa^{-2} f(\cdot / \kappa)}-1}, U_{\kappa}\left(\sqrt{e^{\kappa^{-2} f(\cdot / \kappa)}-1} K_{\kappa \Lambda}^{\beta} \sqrt{e^{\kappa^{-2} f(\cdot / \kappa)}-1}\right)^{-1} \sqrt{e^{\kappa^{-2} f(\cdot / \kappa)}-1}\right\rangle \\
-\frac{1}{\kappa^{d-2}} \log \operatorname{Det}\left[1-U_{\kappa} \sqrt{e^{\kappa^{-2} f(\cdot / \kappa)}-1} K_{\kappa \Lambda}^{\beta} \sqrt{e^{\kappa^{-2} f(\cdot / \kappa)}-1} U_{\kappa}^{-1}\right] \\
=\left(\rho-\rho_{c}\right)\left\langle\sqrt{f_{\kappa}^{(+)}},\left(1-\sqrt{f_{\kappa}^{(+)}} \kappa^{-2} K_{\Lambda}^{\beta / \kappa^{2}} \sqrt{f_{\kappa}^{(+)}}\right)^{-1} \sqrt{f_{\kappa}^{(+)}}\right\rangle \\
+\frac{1}{\kappa^{d}} \operatorname{Tr}\left[f_{\kappa}^{(+)} K_{\Lambda}^{\beta / \kappa^{2}}\right]-\frac{1}{\kappa^{d-2}} \log \operatorname{Det}_{2}\left[1-f_{\kappa}^{(+)} \kappa^{-2} K_{\Lambda}^{\beta / \kappa^{2}}\right] .
\end{gathered}
$$

Applying Lemma 3.7, 3.5 to the first term, Lemma 3.8, 3.5 to the second term and Lemma 3.9 to the third term, we get

$$
P(1)=\left(\rho-\rho_{c}\right)\left\langle\sqrt{f},\left[1-\sqrt{f}(-\beta \Delta)_{\Lambda}^{-1} \sqrt{f}\right]^{-1} \sqrt{f}\right\rangle+\rho_{c} \int_{\mathbb{R}^{d}} f(x) d x .
$$

Then the Lemma 4.6 proves the case $\left\|\sqrt{f}(-\beta \Delta)_{\Lambda}^{-1} \sqrt{f}\right\|<1$.

When $\left\|\sqrt{f}(-\beta \Delta)_{\Lambda}^{-1} \sqrt{f}\right\|>1$, we apply $U_{\kappa}$ and Lemmas 3.7, 3.3 to find that

$$
\left\|\sqrt{e^{f(\cdot / \kappa) / \kappa^{2}}-1} K_{\kappa \Lambda}^{\beta} \sqrt{e^{f(\cdot / \kappa) / \kappa^{2}}-1}\right\| \geqslant 1
$$

for $\kappa$ large enough. Therefore, we get from Theorem 2.1(2),(4) that

$$
\lim _{\kappa \rightarrow \infty} \int_{Q\left(\mathbb{R}^{d}\right)} e^{\left\langle f(\cdot / \kappa) / \kappa^{2}, \xi\right\rangle} \nu_{\rho}(d \xi)=\infty
$$

When $\left\|\sqrt{f}(-\beta \Delta)_{\Lambda}^{-1} \sqrt{f}\right\|=1$, then applying Lemma 3.7 and transformation $U_{\kappa}$, we find for large $\kappa$ the estimate:

$$
\left\|\sqrt{e^{f(\cdot / \kappa) / \kappa^{2}}-1} K_{\kappa \Lambda}^{\beta} \sqrt{e^{f(\cdot / \kappa) / \kappa^{2}}-1}\right\|=\left\|\sqrt{f_{\kappa}^{(+)}} \kappa^{-2} K_{\Lambda}^{\beta / \kappa^{2}} \sqrt{f_{\kappa}^{(+)}}\right\| \geqslant 1-c \kappa^{-2} .
$$

In fact, it is enough to consider the case where the above quantity is smaller than 1 . In this case Lemmas 3.5, 3.7 and 3.1 yield

$$
\begin{aligned}
& \left|\left\langle\sqrt{f_{\kappa}^{(+)}},\left(\sqrt{f_{\kappa}^{(+)}} \kappa^{-2} K_{\Lambda}^{\beta / \kappa^{2}} \sqrt{f_{\kappa}^{(+)}}\right)^{n} \sqrt{f_{\kappa}^{(+)}}\right\rangle-\left\langle\sqrt{f},\left(\sqrt{f}(-\beta \Delta)_{\Lambda}^{-1} \sqrt{f}\right)^{n} \sqrt{f}\right\rangle\right| \\
\leqslant & \left|\left\langle\sqrt{f_{\kappa}^{(+)}}-\sqrt{f},\left(\sqrt{f_{\kappa}^{(+)}} \kappa^{-2} K_{\Lambda}^{\beta / \kappa^{2}} \sqrt{f_{\kappa}^{(+)}}\right)^{n} \sqrt{f_{\kappa}^{(+)}}\right\rangle\right| \\
& +\left|\left\langle\sqrt{f},\left(\sqrt{f_{\kappa}^{(+)}} \kappa^{-2} K_{\Lambda}^{\beta / \kappa^{2}} \sqrt{f_{\kappa}^{(+)}}\right)^{n}\left(\sqrt{f_{\kappa}^{(+)}}-\sqrt{f}\right)\right\rangle\right| \\
& +\left|\left\langle\sqrt{f},\left\{\left(\sqrt{f_{\kappa}^{(+)}} \kappa^{-2} K_{\Lambda}^{\beta / \kappa^{2}} \sqrt{f_{\kappa}^{(+)}}\right)^{n}-\left(\sqrt{f}(-\beta \Delta)_{\Lambda}^{-1} \sqrt{f}\right)^{n}\right\} \sqrt{f}\right\rangle\right| \\
\leqslant & \frac{\|f\|_{\infty}}{\kappa^{2}}\left(1+e^{\|f\|_{\infty} / \kappa^{2}}\right) e^{\|f\|_{\infty} / \kappa^{2}}\langle\sqrt{f}, \sqrt{f}\rangle \\
& +n\langle\sqrt{f}, \sqrt{f}\rangle\left\|\sqrt{f_{\kappa}^{(+)}} \kappa^{-2} K_{\Lambda}^{\beta / \kappa^{2}} \sqrt{f_{\kappa}^{(+)}}-\sqrt{f}(-\beta \Delta)_{\Lambda}^{-1} \sqrt{f}\right\| \\
\leqslant & c \frac{n+1}{\kappa^{2}} .
\end{aligned}
$$


This estimate together with Theorem 2.1(2), give the limit:

$$
\begin{aligned}
& \frac{1}{\kappa^{d-2}} \log \int_{Q\left(\mathbb{R}^{d}\right)} e^{\left\langle f(\cdot / \kappa) / \kappa^{2}, \xi\right\rangle} \mu_{K^{\beta},\left(\rho-\rho_{c}\right)}(d \xi) \\
= & \frac{\rho-\rho_{c}}{\kappa^{d-2}}\left\langle\sqrt{e^{f(\cdot / \kappa) / \kappa^{2}}-1},\left(1-\sqrt{e^{f(\cdot / \kappa) / \kappa^{2}}-1} K_{\kappa \Lambda}^{\beta} \sqrt{e^{f(\cdot / \kappa) / \kappa^{2}}-1}\right)^{-1} \sqrt{e^{f(\cdot / \kappa) / \kappa^{2}}-1}\right\rangle \\
= & \left(\rho-\rho_{c}\right) \sum_{n=0}^{\infty}\left\langle\sqrt{f_{\kappa}^{(+)}},\left(\sqrt{f_{\kappa}^{(+)}} \kappa^{-2} K_{\Lambda}^{\beta / \kappa^{2}} \sqrt{f_{\kappa}^{(+)}}\right)^{n} \sqrt{f_{\kappa}^{(+)}}\right\rangle \\
\geqslant & \left(\rho-\rho_{c}\right) \sum_{n=0}^{\infty}\left\{\left\langle\sqrt{f},\left(\sqrt{f}(-\beta \Delta)_{\Lambda}^{-1} \sqrt{f}\right)^{n} \sqrt{f}\right\rangle\right. \\
& \left.-\left|\left\langle\sqrt{f_{\kappa}^{(+)}},\left(\sqrt{f_{\kappa}^{(+)}} \kappa^{-2} K_{\Lambda}^{\beta / \kappa^{2}} \sqrt{f_{\kappa}^{(+)}}\right)^{n} \sqrt{f_{\kappa}^{(+)}}\right\rangle-\left\langle\sqrt{f},\left(\sqrt{f}(-\beta \Delta)_{\Lambda}^{-1} \sqrt{f}\right)^{n} \sqrt{f}\right\rangle\right|\right\} \vee 0 \\
\geqslant & \left(\rho-\rho_{c}\right) \sum_{n=0}^{\infty}\left\{|\langle\varphi, \sqrt{f}\rangle|^{2}-c \frac{n+1}{\kappa^{2}}\right\} \vee 0 \geqslant\left(\rho-\rho_{c}\right) \frac{|\langle\varphi, \sqrt{f}\rangle|^{4} \kappa^{2}}{2 c} \rightarrow \infty .
\end{aligned}
$$

when $\kappa \rightarrow \infty$. Here we applied $U_{\kappa}$ in the second equality, and then the fact that $\varphi$ is the eigenfunction of the operator $\sqrt{f}(-\beta \Delta)_{\Lambda}^{-1} \sqrt{f}$ with the largest eigenvalue 1 . Note that $\langle\sqrt{f}, \varphi\rangle>0$. In fact, since the integral kernel of this operator is positive on the set $\{f>0\}$, one gets: $\varphi>0$ a.e. on $\{f>0\}$, c.f. Remark $4.8(2)$.

The corresponding estimate for $\mu_{K^{\beta}}^{(d e t)}$ is straightforward.

Recall that the Fenchel-Legendre transformation of the function $P$ has the form:

$$
I(s):=\sup _{s \in \mathbb{R}}(s t-P(t))
$$

By virtue of the Gärtner-Ellis theorem (see e.g. [DZ]), we obtained the following large deviation principle.

Theorem 4.9 (Large Deviation Principle) The random variable $\left\langle f(\cdot / \kappa) / \kappa^{2}, \xi\right\rangle$ satisfies in the condensation regime $\rho>\rho_{c}$ the large deviation principle with a bona fide rate function $I$ :

$$
\limsup _{\kappa \rightarrow \infty} \frac{1}{\kappa^{d-2}} \log \nu_{\rho}\left[\left\langle\frac{1}{\kappa^{d}} f\left(\frac{\dot{\kappa}}{\kappa}\right), \xi\right\rangle \in F\right] \leqslant-\inf _{s \in F} I(s) \quad \text { forarbitraryclosed } F \subset \mathbb{R}
$$

and

$$
\liminf _{\kappa \rightarrow \infty} \frac{1}{\kappa^{d-2}} \log \nu_{\rho}\left[\left\langle\frac{1}{\kappa^{d}} f\left(\frac{\dot{\kappa}}{\kappa}\right), \xi\right\rangle \in G\right] \geqslant-\inf _{s \in G} I(s) \quad \text { forarbitraryopen } G \subset \mathbb{R} .
$$

Remark 4.10 Note that contribution of the point processus $\mu_{K^{\beta}}^{(d e t)}$ to the large deviation property is in a sense marginal, since it only shifts the variable s of the rate function I see (4.4). Taking into account the central limit theorem, we see that the characteristic feature of the limit theorems for the ideal boson gas in the presence of the Bose-Einstein condensation is reflected by the convolution with a nontrivial component $\mu_{K^{\beta}, \rho}$. This gives $\nu_{\rho}=\mu_{K^{\beta}}^{(\text {det })} * \mu_{K^{\beta},\left(\rho-\rho_{c}\right)}$. 


\section{Conclusion}

To compare our results for the case: $\rho>\rho_{c}$ (BEC), we would like to mention here the corresponding results for the case $\rho<\rho_{c}$ (normal phase without condensation).

Let us put $K_{z}:=z G^{\beta}\left(1-z G^{\beta}\right)^{-1}$ with $z \in(0,1)$, which satisfies $\rho=K_{z}(x, x)$ and $\nu_{\rho}=\mu_{K_{z}}^{(\text {det })}$. Then for $\rho<\rho_{c}$ our theorems take the following form, see [LLS, GLM, ShTa]:

Theorem 5.1 (The law of large number) For $\kappa \rightarrow \infty$ one has

$$
\frac{1}{\kappa^{d}}\langle f(\cdot / \kappa), \xi\rangle \longrightarrow \rho \int_{\mathbb{R}^{d}} f(x) d x \quad \text { in } \quad L^{2}\left(Q\left(\mathbb{R}^{d}\right), \nu_{\rho}\right)
$$

Theorem 5.2 (The central limit theorem) For the random variables

$$
Z_{\kappa}=\frac{\langle f(\cdot / \kappa), \xi\rangle-\kappa^{d} \rho \int_{\mathbb{R}^{d}} f(x) d x}{\sqrt{K_{z}(x, x)+K_{z}^{2}(x, x)}\|f\|_{2} \kappa^{d / 2}},
$$

one gets the limit:

$$
\lim _{\kappa \rightarrow \infty} \int_{Q\left(\mathbb{R}^{d}\right)} e^{i t Z_{\kappa}} \nu_{\rho}(d \xi)=e^{-t^{2} / 2} .
$$

Theorem 5.3 (Large deviation principle) There exists a certain bona fide rate convex function $I^{\prime}: \mathbb{R} \mapsto[0,+\infty]$, such that

$$
\limsup _{\kappa \rightarrow \infty} \frac{1}{\kappa^{d}} \log \nu_{\rho}\left(\frac{1}{\kappa^{d}}\langle f(\cdot / \kappa), \xi\rangle \in F\right) \leqslant-\inf _{s \in F} I^{\prime}(s) \quad \text { foranyclosed } F \subset \mathbb{R}
$$

and

$$
\liminf _{\kappa \rightarrow \infty} \frac{1}{\kappa^{d}} \log \nu_{\rho}\left(\frac{1}{\kappa^{d}}\langle f(\cdot / \kappa), \xi\rangle \in G\right) \geqslant-\inf _{s \in G} I^{\prime}(s) \quad \text { foranyopen } G \subset \mathbb{R}
$$

hold.

We may summarize the difference between Theorems 5.15 .3 and Theorems 1.1 1.3 as follows. Let

$$
D_{\kappa}=\frac{1}{\kappa^{d}}\langle f(\cdot / \kappa), \xi\rangle
$$

be the a random variable corresponding to empirical "density" of particles localised in the region of length scale $\kappa$.

For the BEC case one gets:

(i) The random variable $D_{\kappa}$ converges for $\kappa \rightarrow \infty$ to its expectation value $m=$ $\rho \int_{\mathbb{R}^{d}} f(x) d x$ in mean. 
(ii) The law of the random variable $\kappa^{(d-2) / 2}\left(D_{\kappa}-m\right)$ converges to normal distribution as $\kappa \rightarrow \infty$.

(iii) The law of the random variable $D_{\kappa}$ manifests a large deviation property with parameter $\kappa^{d-2}$.

For the normal phase:

(i) also holds; (ii) holds but for $\kappa^{d / 2}\left(D_{\kappa}-m\right)$, instead of $\kappa^{(d-2) / 2}\left(D_{\kappa}-m\right)$; and (iii) holds with the order $\kappa^{d}$, instead of $\kappa^{d-2}$.

The comparison shows that there are differences in deviation of density fluctuation between the BEC and the non-BEC states of ideal boson gases, which reminds the large deviation properties for two-phase classical systems, for example lattice spin models, see e.g. $[\mathrm{P}$. The specificity of the BEC is that it is a quantum phase transition with particular quantum fluctuations [LePu, [ZB].

\section{Acknowledgments}

H.T. thanks JSPS for the financial support under the Grant-in-Aid for Scientific Research (C) 20540162 and Centre de Physique Théorique, Luminy-Marseille for hospitality. V.A.Z. is grateful to Mathematical Department of the Kanazawa University for a warm hospitality and for financial support.

\section{References}

[BrRo] O. Bratteli and D. W. Robinson, Operator algebras and quantum statistical mechanics II (Springer-Verlag, Berlin, 1996)

[BZ] J.-B.Bru and V.A. Zagrebnov, Large Deviations in the Superstable Weakly Imperfect Bose-Gas, J.Stat.Phys. 133, 379-400 (2008).

[DV] D. J. Daley and D. Vere-Jones, An Introduction to the Theory of Point Processes (Springer, Berlin, 1988).

[DZ] A. Dembo and O. Zeitouni, Large deviations techniques and Applications 2nd. ed. (Springer, Berlin, 1998).

[deS-Z] Ph. de Smedt and V.A. Zagrebnov, Van der Waals Limit of an Interacting Bose Gas in a Weak External Field, Phys. Rev. A35, 4763-4769 (1987).

[DGPS] F. Dalfovo, S.Giorgini, L. P. Pitaevskii and S. Stringari, Theory of BoseEinstein condensation in trapped gases, Rev. Mod. Phys. 71, 463-512 (1999).

[E] N. Eisenbaum, A Cox process involved in the Bose-Einstein condensation, Annales Henri Poincaré 9, 1123-1140 (2008).

[F] K.-H. Fichtner, On the position distribution of the ideal Bose gas. Math. Nachr. 151, 59-67 (1991) 
[Fr] W. Freudenberg, Characterization of states of infinite boson systems. II: On the existence of the conditional reduced density matrix. Commun. Math. Phys. 137, 461-472 (1991)

[FFr1] K.-H. Fichtner and W. Freudenberg, Point processes and the position distribution of infinite boson systems. J. Stat. Phys. 47, 959-978 (1987)

[FFr2] K.-H. Fichtner and W. Freudenberg, Characterization of states of infinite boson systems. I: On the construction of states of boson systems. Commun. Math. Phys. 137, 315-357 (1991)

[GLM] G. Gallavotti, J.L. Lebowitz and V. Masropietro, Large Deviations in Rarified Quantum Gases, J.Stat.Phys. 108, 831-861 (2002).

[Ka] T. Kato, Perturbation Theory for Linear Operators (Springer-Verlag, Heidelberg-Berlin, 1966).

[LVZ] J. Lauwers, A. Verbeure and V. A. Zagrebnov, Bose-Einstein Condensation for Homogeneous Interactig System with a One-Particle Spaectral Gap, J. Stat. Phys. 112, 397-420 (2003).

[LLS] J.L. Lebowitz, M. Lenci and H. Spohn, Large Deviations for Ideal Quantum Systems, J. Math. Phys. 41, 1224-1243 (2000).

[LePu] J. Lewis and J. V. Pulè, The Equilibrium States of the Free Boson Gas, Commun. Math. Phys. 36, 1-18 (1974) .

[LSSY] E. H. Lieb, R. Seiringer, J. P. Solovej and J. Yngvason, The Mathematics of the Bose Gas and its Condensation (Birkhaeuser, Basel, 2005).

[Ly] E. Lytvynov, Fermion and boson random point processes as particle distributions of infinite free Fermi and Bose gases of finite density, Rev. Math. Phys. 14, 1073-1098 (2002).

[M1] O. Macchi, The coincidence approach to stochastic point processes, Adv. Appl. Prob. 7, 83-122 (1975).

[M2] O. Macchi, The fermion process-a model of stochastic point process with repulsive points, pp.391-398 in Transactions of the Seventh Prague Conference on Information Theory, Statistical Decision Functions, Random Processes and of the Eighth European Meeting of Statisticians (Tech. Univ. Prague, 1974), Vol. A (Reidel, Dordrecht, 1977)

[MaVe] J. Manuceau and A. Verbeure, Quasi-free states of the CCR-algebra and Bogoliubov transformations, Commun. Math. Phys. 9, 125-131 (1977).

[PeSm] C.J. Pethick and H. Smith, Bose-Einstein Condensation in Dilute Gases (Cambridge University Press, Cambridge, (2002)). 
[P] A. Pisztora, Surface order large deviations for Ising, Potts and percolation models, Probab. Theory Rel. Fields 104, 427-466 (1996).

$[\mathrm{Pu}] \quad$ J. V. Pulè, The free boson gas in a weak external potential, J. Math. Phys. 24, 138-142 (1983).

[RSIV] M. Reed and B. Simon, Methods of Modern Mathematical Physics, vol. IV: Analysis of Operatyors (Academic Press, London, 1978).

[S] A. Soshnikov, Determinantal random point processes. Russian Math. Surveys 55, 923-975 (2000).

[ShTa] T. Shirai and Y. Takahashi, Random point fields associated with certain Fredholm determinants I: fermion, Poisson and boson point processes, J. Funct. Anal. 205, 414-463 (2003).

[TI1] H. Tamura and K.R. Ito, A Canonical Ensemble Approach to the Fermion/Boson Random Point Processes and its Applications, Commun. Math. Phys. 263, 353-380 (2006).

[TI2] H. Tamura and K.R. Ito, A Random Point Field related to Bose-Einstein Condensation, J. Funct. Anal. 243, 207-231 (2007).

[TI3] H. Tamura and K.R. Ito, Random Point Fields for Para-Particles of Any Order, J. Math. Phys. 48 023301, 14 pages (2007).

[TZ] H. Tamura and V.A. Zagrebnov, Mean-Field Interacting Boson Random Point Fields in Weak Harmonic Traps, J. Math. Phys. 50, 023301, 28 pages (2009) .

[VVZ] L. Vandevenne, A. Verbeure and V. A. Zagrebnov, Equilibrium states for the Bose gas, J.Math.Phys. 45, 1606-1622 (2004).

[V] D. Vere-Jones, A generalization of permanents and determinants, Linear Algebra Appl. 111, 119-124 (1988).

[Z] V.A. Zagrebnov, Topics in the Theory of Gibbs Semigroups. Leuven Notes in Mathematical and Theoretical Physics Vol. 10 (Leuven University Press, Leuven 2002).

[ZB] V.A. Zagrebnov and J.-B. Bru, The Bogoliubov model of weakly imperfect Bose gas, Phys. Rep. 350, 291-434 (2001). 